\title{
Similarity Measures of Sequence of Fuzzy Numbers and Fuzzy Risk Analysis
}

\author{
Zarife Zararsız \\ Department of Mathematics, Faculty of Art and Science, University of Nevşehir Hacı Bektaş Veli, 50300 Nevşehir, Turkey \\ Correspondence should be addressed to Zarife Zararsız; zarifezararsiz@nevsehir.edu.tr
}

Received 18 May 2015; Revised 21 July 2015; Accepted 22 July 2015

Academic Editor: Soheil Salahshour

Copyright (c) 2015 Zarife Zararsiz. This is an open access article distributed under the Creative Commons Attribution License, which permits unrestricted use, distribution, and reproduction in any medium, provided the original work is properly cited.

\begin{abstract}
We present the methods to evaluate the similarity measures between sequence of triangular fuzzy numbers for making contributions to fuzzy risk analysis. Firstly, we calculate the COG (center of gravity) points of sequence of triangular fuzzy numbers. After, we present the methods to measure the degree of similarity between sequence of triangular fuzzy numbers. In addition, we give an example to compare the methods mentioned in the text. Furthermore, in this paper, we deal with the $\left(t_{1}, t_{2}\right)$ type fuzzy number. By defining the algebraic operations on the $\left(t_{1}, t_{2}\right)$ type fuzzy numbers we can solve the equations in the form $x+u_{\left(t_{1}, t_{2}\right)}=v_{\left(t_{1}, t_{2}\right)}$, where $u_{\left(t_{1}, t_{2}\right)}$ and $v_{\left(t_{1}, t_{2}\right)}$ are fuzzy number. By this way, we can build an algebraic structure on fuzzy numbers. Additionally, the generalized difference sequence spaces of triangular fuzzy numbers $\left[\ell_{\infty}\left(F_{t}\right)\right]_{\mathscr{B}(\hat{r}, \hat{S})},\left[c\left(F_{t}\right)\right]_{\mathscr{B}(\hat{r}, \hat{S})}$, and $\left[c_{0}\left(F_{t}\right)\right]_{\mathscr{B}(\hat{r}, \hat{S})}$, consisting of all sequences $u^{*}=\left(u_{\left(t_{1}, t_{2}\right)}^{k}\right)$ such that $\mathscr{B}(\widehat{r}, \widehat{s}) u^{*}$ is in the spaces $\ell_{\infty}\left(F_{t}\right), c\left(F_{t}\right)$, and $c_{0}\left(F_{t}\right)$, have been constructed, respectively. Furthermore, some classes of matrix transformations from the space $\left[c\left(F_{t}\right)\right]_{\mathscr{B}(\widehat{r}, \hat{s})}$ and $\mu\left(F_{t}\right)$ to $\mu\left(F_{t}\right)$ and $\left[c\left(F_{t}\right)\right]_{\mathscr{B}(\hat{r}, \hat{s})}$ are characterized, respectively, where $\mu\left(F_{t}\right)$ is any sequence space.
\end{abstract}

\section{Preliminaries, Background, and Notation}

The concept of fuzzy sets and fuzzy set operations were first introduced by [1]. After his innovation many authors have studied various aspects of the fuzzy set theory and its applications, such as fuzzy topological spaces, similarity relations, and fuzzy mathematical programming. Matloka [2] introduced the class of bounded and convergent sequences of fuzzy numbers with respect to the Hausdorff metric. In [3], Nanda has studied the spaces of bounded and convergent sequences of fuzzy numbers and shown that these spaces are complete metric spaces.

Measuring the similarity between sequences of fuzzy numbers is very important subject of fuzzy decision making $[4,5]$ and fuzzy risk analysis [6]. In [7], a method for fuzzy risk analysis based on similarity measures of generalized fuzzy numbers is given. In [8], a method for measuring the rate of aggregative risk in software development is presented. Recently, some methods have been introduced to calculate the degree of similarity between fuzzy numbers [4-6]. However, these measures cannot determine the degree of similarity between sequences of fuzzy numbers. By generalizing these methods from fuzzy numbers to sequence of triangular fuzzy numbers, we give the methods to evaluate the similarity measures of sequence of triangular fuzzy numbers. In this paper, we also calculate the COG (center of gravity) points of a sequence of triangular fuzzy numbers.

It will not be right to regard this paper as a copy of classic summability theory because both a big generalization and definitions of fuzzy zero are presented in this paper. Therefore, the readers are advised to take these into consideration while reading the paper.

Some important problems on sequence spaces of fuzzy numbers can be ordered as follows: (1) construct a sequence space of fuzzy numbers and compute $\alpha$-dual, $\beta$-dual, and $\gamma$ dual, (2) find some isomorphic spaces of it, (3) give some theorems about matrix transformations on sequence space of fuzzy numbers, and (4) study some inclusion problems and other properties.

In the present paper, we will define matrix domain of sequence spaces of triangular fuzzy numbers and introduce the sequence spaces of fuzzy numbers $\left[\ell_{\infty}\left(F_{t}\right)\right]_{\mathscr{B}(\hat{r}, \hat{s})}$, $\left[c\left(F_{t}\right)\right]_{\mathscr{B}(\hat{r}, \hat{s})}$, and $\left[c_{0}\left(F_{t}\right)\right]_{\mathscr{B}(\hat{r}, \widehat{s})}$. Additionally, we redefine fuzzy identity elements according to addition and multiplication 
for constructing an algebraic structure on the $\left(t_{1}, t_{2}\right)$ type sets of fuzzy numbers.

The rest of our paper is organized as follows.

In Section 2, some basic definitions and theorems related to the fuzzy numbers are given. In Section 3, we have introduced generalized difference sequence space of triangular fuzzy numbers and proved some inclusion relations on these sequence spaces. It is also established in Section 3 that the sequence spaces of triangular fuzzy numbers showed by $\left[\ell_{\infty}\left(F_{t}\right)\right]_{\mathscr{B}(\widehat{r}, \widehat{s})},\left[c\left(F_{t}\right)\right]_{\mathscr{B}(\hat{r}, \widehat{s})}$, and $\left[c_{0}\left(F_{t}\right)\right]_{\mathscr{B}(\widehat{r}, \widehat{s})}$ are linearly isomorphic to the spaces $\ell_{\infty}\left(F_{t}\right), c\left(F_{t}\right)$, and $c_{0}\left(F_{t}\right)$, respectively. Finally, in Section 3 , it is proved that the spaces $\left[\ell_{\infty}\left(F_{t}\right)\right]_{\mathscr{B}(\hat{r}, \hat{s})},\left[c\left(F_{t}\right)\right]_{\mathscr{B}(\hat{r}, \widehat{s})}$, and $\left[c_{0}\left(F_{t}\right)\right]_{\mathscr{B}(\hat{r}, \hat{s})}$ are complete. Section 4 is devoted to the calculation of the $\beta(r)$ - and $\gamma(r)$-duals of the spaces $\left[c\left(F_{t}\right)\right]_{\mathscr{B}(\hat{r}, \hat{s})}$ and $\left[c_{0}\left(F_{t}\right)\right]_{\mathscr{B}(\hat{r}, \widehat{s})}$. In Section 5, some classes of matrix transformations from the space $\left[c\left(F_{t}\right)\right]_{\mathscr{B}(\hat{r}, \widehat{s})}$ and $\mu\left(F_{t}\right)$ to $\mu\left(F_{t}\right)$ and $\left[c\left(F_{t}\right)\right]_{\mathscr{B}(\hat{r}, \widehat{s})}$ are characterized, respectively, where $\mu\left(F_{t}\right)$ is any sequence space. In Section 6, we review the COG points of a sequence of fuzzy numbers [7] and existing similarity measures of fuzzy numbers [4-7]. In Section 7, we generalize the use of similarity measure methods to sequence of triangular fuzzy numbers. Furthermore, we use an example to compare the similarity measure methods between three sets of sequences of triangular fuzzy numbers. In the final section, we consider the similarity measure methods of sequence of triangular fuzzy numbers to deal with the fuzzy risk analysis problems.

In this section, we recall some of the basic definitions and notions in the theory of fuzzy numbers. Let us suppose that $\mathbb{N}, \mathbb{R}$, and $E_{i}=\left\{\alpha=\left[\alpha^{-}, \alpha^{+}\right]: \alpha^{-} \leq x \leq \alpha^{+}, \alpha^{-}\right.$and $\left.\alpha^{+} \in \mathbb{R}\right\}$ are the set of all positive integers, all real numbers, and all bounded and closed intervals on the real line $\mathbb{R}$, respectively. For $\alpha, \beta \in E_{i}$ define the following metric:

$$
d(\alpha, \beta)=\max \left\{\left|\alpha^{-}-\beta^{-}\right|,\left|\alpha^{+}-\beta^{+}\right|\right\} .
$$

It can easily be seen that $d$ defines a metric on $E_{i}$ and the pair $\left(E_{i}, d\right)$ is a complete metric space [9]. Let $X$ be a nonempty set. According to Zadeh a fuzzy subset of $X$ is a nonempty subset $\{(x, \mathbf{u}(x)): x \in X\}$ of $X \times[0,1]$ for some function $\mathbf{u}: X \rightarrow[0,1][10]$. Consider a function $\mathbf{u}: \mathbb{R} \rightarrow[0,1]$ as a subset of a nonempty base space $\mathbb{R}$ and denote the family of all such functions or fuzzy sets by $E$. Let us suppose that the function $\mathbf{u}$ satisfies the following properties:

(1) $\mathbf{u}$ is normal, that is, there exists an $x_{0} \in \mathbb{R}$ such that $\mathbf{u}\left(x_{0}\right)=1$.

(2) $\mathbf{u}$ is fuzzy convex, that is, for any $x, y \in \mathbb{R}$ and $t \in$ $[0,1], \mathbf{u}[t x+(1-t) y] \geq \min \{\mathbf{u}(x), \mathbf{u}(y)\}$.

(3) $\mathbf{u}$ is upper semicontinuous.

(4) The closure of $\{x \in \mathbb{R}: \mathbf{u}(x)>0\}$, denoted by $\mathbf{u}^{0}$, is compact.

Then, the function $\mathbf{u}$ is called a fuzzy number [11].

The properties (1)-(4) imply that, for each $\alpha \in[0,1]$, the $\alpha$-cut set of the fuzzy number $\mathbf{u}$ defined by $\mathbf{u}(\alpha)=$ $\{x \in \mathbb{R}: \mathbf{u}(x) \geq \alpha\}$ is in $E_{i}$. That is, the equality $\mathbf{u}(\alpha)=\left[\mathbf{u}^{-}(\alpha), \mathbf{u}^{+}(\alpha)\right]$ holds for each $\alpha \in[0,1]$. We denote the set of all fuzzy numbers by $F$. Any vector subspace of $w$, the space of all complex valued sequences, is called a sequence space. We write $\ell_{\infty}, c, c_{0}$, and $\ell_{p}$ for the classical sequence spaces of all bounded, convergent, null, and absolutely $p$-summable sequences, respectively. For simplicity in notation, the summation without limits runs from 0 to $\infty$, $\lim _{k \rightarrow \infty}=\lim _{k}, \sum_{k}^{\infty}=\sum_{k}, \sup _{k \in \mathbb{N}}=\sup _{k}$, through all the text.

Let us consider any triangular $\left(t_{1}, t_{2}\right)$ type fuzzy number $u_{\left(t_{1}, t_{2}\right)}$, as follows. If the function

$$
u_{\left(t_{1}, t_{2}\right)}(x)= \begin{cases}\left(x-\left(u-t_{1}\right)\right) t_{1}^{-1}, & x \in\left[u-t_{1}, u\right] \\ \left(\left(u+t_{2}\right)-x\right) t_{2}^{-1}, & x \in\left[u, u+t_{2}\right] \\ 0, & \text { otherwise }\end{cases}
$$

is the membership function of the triangular fuzzy number $u_{\left(t_{1}, t_{2}\right)}$, then $u_{\left(t_{1}, t_{2}\right)}$ can be represented with the notation

$$
u_{\left(t_{1}, t_{2}\right)}=\left(u-t_{1}, u, u+t_{2}\right) \text {, }
$$

where $t_{1}, t_{2} \in \mathbb{R}$ and $t_{1} \leq t_{2}$. If $t_{1}=t_{2}$, then the fuzzy number $u_{\left(t_{1}, t_{2}\right)}$ is called symmetric fuzzy number and if $t_{1}=$ $t_{2}=0$, then $u_{\left(t_{1}, t_{2}\right)}$ is a real number. In general, the fuzzy number $u_{\left(t_{1}, t_{2}\right)}$ is called $\left(t_{1}, t_{2}\right)$-type fuzzy number. After here, we deal with the set of triangular $\left(t_{1}, t_{2}\right)$-type fuzzy numbers represented as $F_{t}$ which includes the classic triangular fuzzy numbers, through the text. Any element of the set $F_{t}$ will be denoted by $u_{\left(t_{1}, t_{2}\right)}$. Additionally, for brevity, we call triangular $\left(t_{1}, t_{2}\right)$-type fuzzy numbers triangular fuzzy numbers.

It means that, set of all $\left(t_{1}, t_{2}\right)$-type fuzzy number is defined as in the following:

$$
F_{t}=\left\{\left(u-t_{1}, u, u+t_{2}\right): t_{1}, t_{2}, u \in \mathbb{R}, t_{1} \leq t_{2}\right\} .
$$

The notations $u-t_{1}, u, u+t_{2}$ are called first, middle, and end points of triangular fuzzy number $u_{\left(t_{1}, t_{2}\right)}$, respectively. In addition to this, the notation $u$ means that the height of the fuzzy number $u_{\left(t_{1}, t_{2}\right)}$ is 1 at the point $u$. For every $t_{1}, t_{2} \in \mathbb{R}$, the sets $F_{t}$ are different from each other and every element in the form $u_{\left(t_{1}, t_{2}\right)}$ of these sets belongs to $F_{t}$. Another mean of the (4) is that for every $t_{1}, t_{2} \in \mathbb{R}$, there is no unique set of fuzzy numbers. Furthermore, there are infinitely-many sets of fuzzy numbers and these sets are different from each other according to structure of their elements. So, we can use the most appropriate one of these sets for our problem. In this study, we will take $t_{1}, t_{2} \in[0,1)$.

Sometimes, the representation of fuzzy numbers with $\alpha$ cut sets induces errors according to algebraic operations. For example, if $\mathbf{v}$ is any fuzzy number, then $\mathbf{v}-\mathbf{v}=\left[\mathbf{v}^{-}(\alpha), \mathbf{v}^{+}(\alpha)\right]-$ $\left[\mathbf{v}^{-}(\alpha), \mathbf{v}^{+}(\alpha)\right]=\left[\mathbf{v}^{-}(\alpha)-\mathbf{v}^{+}(\alpha), \mathbf{v}^{+}(\alpha)-\mathbf{v}^{-}(\alpha)\right], \alpha \in[0,1]$, is not equal to zero as expected in the classic mean. For avoiding this kind of problems we define fuzzy zero represented by $\theta$ as in the following section. 


\section{Algebraic Structure of the Set $F_{t}$}

Let $u_{\left(t_{1}, t_{2}\right)}, v_{\left(t_{1}, t_{2}\right)} \in F_{t}$ and $\alpha \in \mathbb{R}$. Let us define addition, substraction, multiplication, division, and scalar multiplication on $F_{t}$, respectively, as follows:

$$
\begin{aligned}
u_{\left(t_{1}, t_{2}\right)} \oplus v_{\left(t_{1}, t_{2}\right)} & =\left(u-t_{1}, u, u+t_{2}\right) \oplus\left(v-t_{1}, v, v+t_{2}\right) \\
& =\left(u+v-t_{1}, u+v, u+v+t_{2}\right), \\
u_{\left(t_{1}, t_{2}\right)} \ominus v_{\left(t_{1}, t_{2}\right)} & =\left(u-t_{1}, u, u+t_{2}\right) \ominus\left(v-t_{1}, v, v+t_{2}\right) \\
& =\left(u-v-t_{1}, u-v, u-v+t_{2}\right), \\
u_{\left(t_{1}, t_{2}\right)} \otimes v_{\left(t_{1}, t_{2}\right)} & =\left(u-t_{1}, u, u+t_{2}\right) \otimes\left(v-t_{1}, v, v+t_{2}\right) \\
& =\left(u v-t_{1}, u v, u v+t_{2}\right), \\
u_{\left(t_{1}, t_{2}\right)} \oslash v_{\left(t_{1}, t_{2}\right)} & =\left(u-t_{1}, u, u+t_{2}\right) \oslash\left(v-t_{1}, v, v+t_{2}\right) \\
& =\left(u \div v-t_{1}, u \div v, u \div v+t_{2}\right),
\end{aligned}
$$

where $v_{\left(t_{1}, t_{2}\right)}$ is nonzero fuzzy number and

$$
\begin{aligned}
\alpha \otimes u_{\left(t_{1}, t_{2}\right)} & =\left(\alpha u-t_{1}, \alpha u, \alpha u+t_{2}\right)=\left(r-t_{1}, r, r+t_{2}\right) \\
& =r_{\left(t_{1}, t_{2}\right)} .
\end{aligned}
$$

Let $u_{\left(t_{1}, t_{2}\right)}=\left(u-t_{1}, u, u+t_{2}\right) \in F_{t}$; then $\left(u-t_{1}, u, u+t_{2}\right)+(0-$ $\left.t_{1}, 0,0+t_{2}\right)=\left(u+0-t_{1}, u+0, u+0+t_{2}\right)=\left(u-t_{1}, u, u+t_{2}\right)$. It means that $\theta_{\left(t_{1}, t_{2}\right)}=\left(0-t_{1}, 0,0+t_{2}\right)$ is considered as the identity element of $F_{t}$ according to operation which is given in (5). Therefore, we say that the inverse of the fuzzy number $u_{\left(t_{1}, t_{2}\right)}$ is equal to $-u_{\left(t_{1}, t_{2}\right)}=\left(-u-t_{1},-u,-u+t_{2}\right)$, according to addition and the $-u_{\left(t_{1}, t_{2}\right)}$ determines a fuzzy number. With this idea, we can solve equations in the form $x_{\left(t_{1}, t_{2}\right)}+u_{\left(t_{1}, t_{2}\right)}=$ $\theta_{\left(t_{1}, t_{2}\right)}$. From here fuzzy zeros of the sets $F_{t}$ are as follows:

$$
\theta=\theta_{\left(t_{1}, t_{2}\right)}=\left(0-t_{1}, 0,0+t_{2}\right) .
$$

This says to us that fuzzy zero is different for each element of the set $F_{t}$.

Theorem 1. All sets in the form $F_{t}$ are linear spaces according to algebraic operations (5) and (9), where $t_{1}, t_{2} \in \mathbb{R}$ and $t_{1} \leq t_{2}$.

The second important matter is the topology on the set $F_{t}$. Şengönül [12] has constructed a topology on $F_{t}$ by using the metric $\bar{d}: F_{t} \times F_{t} \rightarrow \mathbb{R}$ defined as follows:

$$
\begin{aligned}
& \bar{d}\left(u_{\left(t_{1}, t_{2}\right)}, v_{\left(t_{1}, t_{2}\right)}\right) \\
& \quad:=\max \left\{\left|u-v-t_{1}\right|,|u-v|,\left|u-v+t_{2}\right|\right\} .
\end{aligned}
$$

We can easily show that the set $F_{t}$ is a complete metric space with the metric $\bar{d}$.

Clearly, the representation (3) for $u_{\left(t_{1}, t_{2}\right)}(x)$ is unique, and on the contrary there is a unique $u_{\left(t_{1}, t_{2}\right)}(x)$ for every $-u_{\left(t_{1}, t_{2}\right)}=$ $\left(-u-t_{1},-u,-u+t_{2}\right)$. We know that, generally in the practical applications, the spread of fuzziness should not be very large. So, the value of $\max \left\{\left|u-v-t_{1}\right|,|u-v|,\left|u-v+t_{2}\right|\right\}$ should be as small as possible. Theoretically, the value " $\max \left\{\left|u-v-t_{1}\right|, \mid u-\right.$ $\left.v|| u-v+,t_{2} \mid\right\}$ should be small" not necessary but it has to be in practical applications. For example, the "approximately 1 " can be taken as $1_{\left(t_{1}, t_{2}\right)}=\left(-4-t_{1}, 1,15+t_{2}\right)$ but in the applications, generally, "approximately 1 " is taken as $1_{\left(t_{1}, t_{2}\right)}=$ $\left(5-t_{1}, 1,5+t_{2}\right),\left(0 \leq t_{1} \leq t_{2}<1\right)$. This choice is more accurate than $1_{\left(t_{1}, t_{2}\right)}=\left(-4-t_{1}, 1,15+t_{2}\right)$.

Furthermore, it must be emphasized that a fuzzy number is determined according to specific processes. For example, let $A$ and $B$ be two different specific systems. Then, if $u$ is "approximately 1 " for the system $A$ then "approximately 1 " may not be in the same sense for another system $B$. So, algebraic properties of the systems $A$ and $B$ are different. This can be explained as follows.

Let us suppose that the spread of left and right fuzziness of every number $u$ is equal to $t$ in $A$. Then fuzzy zero is equal to $(-t, 0, t),(0 \leq t<1)$ for the system $A$ and this fuzzy zero is unique for the system $A$.

The function $f: \mathbb{N} \rightarrow F_{t}, k \rightarrow f(k)=u_{\left(t_{1}, t_{2}\right)}^{k}$ is called a sequence of triangular fuzzy numbers and is represented by $u^{*}=\left(u_{\left(t_{1}, t_{2}\right)}^{k}\right),[13]$.

Let us denote the set of all sequences of triangular fuzzy numbers by $w\left(F_{t}\right)$; that is,

$$
\begin{aligned}
& w\left(F_{t}\right) \\
& \quad:=\left\{u^{*}=\left(u_{\left(t_{1}, t_{2}\right)}^{k}\right): u: \mathbb{N} \longrightarrow F_{t}, u(k)=u_{\left(t_{1}, t_{2}\right)}^{k}\right\},
\end{aligned}
$$

where $u_{t_{1}}^{k} \leq u^{k} \leq u_{t_{2}}^{k}$ and $u_{\left(t_{1}, t_{2}\right)}^{k} \in F_{t}$ for all $k \in \mathbb{N}[14]$.

The $u_{t_{1}}^{k}, u^{k}$, and $u_{t_{2}}^{k}$ are called first, middle, and end points of general term of a sequence of fuzzy numbers, respectively. Each subspace of $w(F)$ is called a sequence space of fuzzy numbers.

We define the classical sets $\ell_{\infty}\left(F_{t}\right), c\left(F_{t}\right), c_{0}\left(F_{t}\right)$, and $\ell_{p}\left(F_{t}\right)$ consisting of the bounded, convergent, null, and absolutely $p$ summable sequences of fuzzy numbers, respectively, similar to [12]; it means that

$$
\begin{aligned}
& \ell_{\infty}\left(F_{t}\right)=\left\{u^{*}=\left(u_{\left(t_{1}, t_{2}\right)}^{k}\right) \in w(F): \sup _{k \in \mathbb{N}} \bar{d}\left(u_{\left(t_{1}, t_{2}\right)}^{k}, \theta\right)\right. \\
& <\infty\} \\
& c\left(F_{t}\right)=\left\{u^{*}=\left(u_{\left(t_{1}, t_{2}\right)}^{k}\right) \in w(F): \lim _{k} \bar{d}\left(u_{\left(t_{1}, t_{2}\right)}^{k}, u_{\left(t_{1}, t_{2}\right)}\right)\right. \\
& =0\} \text {, } \\
& c_{0}\left(F_{t}\right)=\left\{u^{*}=\left(u_{\left(t_{1}, t_{2}\right)}^{k}\right) \in w(F): \lim _{k} \bar{d}\left(u_{\left(t_{1}, t_{2}\right)}^{k}, \theta\right)\right. \\
& =0\} \text {, }
\end{aligned}
$$




$$
\begin{aligned}
& \ell_{p}\left(F_{t}\right)=\left\{u^{*}=\left(u_{\left(t_{1}, t_{2}\right)}^{k}\right) \in w(F): \sum_{k} \bar{d}\left(u_{\left(t_{1}, t_{2}\right)}^{k}, \theta\right)^{p}\right. \\
& \quad<\infty, 1 \leq p<\infty\} .
\end{aligned}
$$

We should emphasize here that the sequence spaces of fuzzy numbers $\ell_{\infty}\left(F_{t}\right), c\left(F_{t}\right), c_{0}\left(F_{t}\right)$, and $\ell_{p}\left(F_{t}\right)$ can be reduced to the classical sequence spaces of real numbers $\ell_{\infty}, c, c_{0}$, and $\ell_{p}$, respectively in the special case $\left(u_{\left(t_{1}, t_{2}\right)}^{k}\right)=\left(u_{(1,1)}^{k}\right)$, where $u_{1}^{k} \in$ $\mathbb{R}$ and $u_{\left(t_{1}, t_{2}\right)}^{k} \in F_{t}$. So, the properties and results related to the sequence spaces of $\ell_{\infty}\left(F_{t}\right), c\left(F_{t}\right), c_{0}\left(F_{t}\right)$, and $\ell_{p}\left(F_{t}\right)$ are more general and more useful than the corresponding implications of the spaces $\ell_{\infty}, c, c_{0}$, and $\ell_{p}$, respectively.

Definition 2. Let $\lambda\left(F_{t}\right) \subset w(F) ; \theta^{*}$ is identity element of $\lambda\left(F_{t}\right)$ according to addition and algebraic operations in the sense of (5) and (9). The function $\|\cdot\|: \lambda\left(F_{t}\right) \rightarrow \mathbb{R}$ is called norm on the set $\lambda\left(F_{t}\right)$ if it has the following properties:

(9) $\left\|u^{*}\right\|=0 \Leftrightarrow u^{*}=\theta^{*}$.

(10) $\left\|\alpha u^{*}\right\|=|\alpha|\left\|u^{*}\right\|, \alpha \in \mathbb{R}$.

(11) $\left\|u^{*}+v^{*}\right\| \leq\left\|u^{*}\right\|+\left\|v^{*}\right\|$.

If the function $\|\cdot\|: \lambda\left(F_{t}\right) \rightarrow \mathbb{R}$ satisfies (9)-(11) then $\lambda\left(F_{t}\right)$ is called normed sequence space of the triangular fuzzy numbers. If $\lambda\left(F_{t}\right)$ is complete with respect to the norm $\|\cdot\|$ then $\lambda\left(F_{t}\right)$ is called complete normed sequence space of the triangular fuzzy numbers.

Lemma 3. The sets $c\left(F_{t}\right), c_{0}\left(F_{t}\right)$, and $\ell_{\infty}\left(F_{t}\right)$ are complete normed sequence spaces with the norm defined as follows:

$$
\begin{gathered}
\left\|u^{*}\right\|=\sup _{k} \max \left\{\left|u_{\left(t_{1}, t_{2}\right)}^{k}-v_{\left(t_{1}, t_{2}\right)}^{k}-t_{1}\right|,\right. \\
\left.\left|u_{\left(t_{1}, t_{2}\right)}^{k}-v_{\left(t_{1}, t_{2}\right)}^{k}\right|,\left|u_{\left(t_{1}, t_{2}\right)}^{k}-v_{\left(t_{1}, t_{2}\right)}^{k}+t_{2}\right|\right\},
\end{gathered}
$$

where $u^{*}$ is in any sets of $\left\{c\left(F_{t}\right), c_{0}\left(F_{t}\right), \ell_{\infty}\left(F_{t}\right)\right\}$.

Now, let $\lambda\left(F_{t}\right)$ and $\mu\left(F_{t}\right)$ be two spaces of triangular fuzzy valued sequences and let $\mathscr{A}=\left(a_{n k}\right)$ be an infinite matrix of positive real numbers $a_{n k}$, where $n, k \in \mathbb{N}$. Then, we say that $\mathscr{A}$ defines a real-matrix mapping from $\lambda\left(F_{t}\right)$ to $\mu\left(F_{t}\right)$ and we denote it by writing $\mathscr{A}: \lambda\left(F_{t}\right) \rightarrow \mu\left(F_{t}\right)$, if for every sequence $u^{*}=\left(u_{\left(t_{1}, t_{2}\right)}^{k}\right) \in \lambda\left(F_{t}\right)$ the sequence $\mathscr{A} u^{*}=\left\{\left(\mathscr{A} u_{\left(t_{1}, t_{2}\right)}\right)^{n}\right\}$ is in $\mu\left(F_{t}\right)$ where

$$
\begin{aligned}
\left(\mathscr{A} u_{\left(t_{1}, t_{2}\right)}\right)^{n} & =\sum_{k} a_{n k} u_{\left(t_{1}, t_{2}\right)}^{k} \\
& =\left(\sum_{k} a_{n k} u_{t_{1}}^{k}, \sum_{k} a_{n k} u^{k}, \sum_{k} a_{n k} u_{t_{2}}^{k}\right)
\end{aligned}
$$

and the series $\sum_{k} a_{n k} u_{t_{1}}^{k}, \sum_{k} a_{n k} u^{k}, \sum_{k} a_{n k} u_{t_{2}}^{k}$ are convergent for all $n \in \mathbb{N}$. By $\left(\lambda\left(F_{t}\right): \mu\left(F_{t}\right)\right)$, we denote the class of matrices $\mathscr{A}$ such that $\mathscr{A}: \lambda\left(F_{t}\right) \rightarrow \mu\left(F_{t}\right)$. Thus, $\mathscr{A} \in\left(\lambda\left(F_{t}\right)\right.$ : $\left.\mu\left(F_{t}\right)\right)$ if and only if the series on the right side of (15) are convergent for each $n \in \mathbb{N}$ and every $u \in \lambda\left(F_{t}\right)$, and we have $\mathscr{A} u^{*}=\left\{\left(\mathscr{A} u_{\left(t_{1}, t_{2}\right)}\right)^{n}\right\}_{n \in \mathbb{N}} \in \mu\left(F_{t}\right)$ for all $u^{*} \in \lambda\left(F_{t}\right)$.

Let $\lambda\left(F_{t}\right)$ be a sequence space of triangular fuzzy numbers. Then, the set $\left[\lambda\left(F_{t}\right)\right]_{\mathscr{A}}$ of sequences of triangular fuzzy numbers, defined as follows, is called the domain of an infinite matrix $\mathscr{A}$ in $\lambda\left(F_{t}\right)$ :

$$
\begin{aligned}
& {\left[\lambda\left(F_{t}\right)\right]_{\mathscr{A}}} \\
& \quad=\left\{u^{*}=\left(u_{\left(t_{1}, t_{2}\right)}^{k}\right) \in w\left(F_{t}\right): \mathscr{A} u_{\left(t_{1}, t_{2}\right)}^{k} \in \lambda\left(F_{t}\right)\right\} .
\end{aligned}
$$

Let $r^{k}, s^{k}$ be nonzero real numbers for each $k \in \mathbb{N}$ and define the band matrix $\mathscr{B}(\widehat{r}, \widehat{s})=\left\{b_{n k}\left(r^{k}, s^{k}\right)\right\}_{n, k=0}^{\infty}$ by

$$
b_{n k}\left(r^{k}, s^{k}\right)= \begin{cases}r^{k}, & k=n \\ s^{k}, & k=n-1 \\ 0, & 0 \leq k<n-1 \text { or } k>n\end{cases}
$$

$(k, n \in \mathbb{N})$.

Here, $\widehat{r}=\left(r^{k}\right)$ and $\widehat{s}=\left(s^{k}\right)$ are the convergent sequences.

It is easy to calculate that the inverse $\mathscr{B}^{-1}(\widehat{r}, \widehat{s})=$ $\left(a_{n k}(\widehat{r}, \widehat{s})\right)$ of the generalized difference matrix $\mathscr{B}(\widehat{r}, \widehat{s})$ is given by

$$
a_{n k}(\widehat{r}, \widehat{s})=\left\{\begin{array}{ll}
\frac{(-1)^{n-k}}{r^{n}} \prod_{j=k}^{n-1} \frac{s^{j}}{r^{j}}, & 0 \leq k \leq n \\
0, & k>n ;
\end{array} \quad(k, n \in \mathbb{N}) .\right.
$$

\section{The Generalized Difference Sequence Spaces

$$
\left[\ell_{\infty}\left(F_{t}\right)\right]_{\mathscr{B}(\hat{r}, \hat{s})},\left[c\left(F_{t}\right)\right]_{\mathscr{B}(\hat{r}, \hat{s})} \text {, and }\left[c_{0}\left(F_{t}\right)\right]_{\mathscr{B}(\hat{r}, \hat{s})}
$$

In this section, we wish to introduce the $\left[\ell_{\infty}\left(F_{t}\right)\right]_{\mathscr{B}(\widehat{r}, \widehat{s})}$, $\left[c\left(F_{t}\right)\right]_{\mathscr{B}(\widehat{r}, \hat{s})}$, and $\left[c_{0}\left(F_{t}\right)\right]_{\mathscr{B}(\widehat{r}, \widehat{s})}$ spaces as the set of all sequences such that $\mathscr{B}(\widehat{r}, \widehat{s})$-transforms of them are in the spaces $\ell_{\infty}\left(F_{t}\right)$, $c\left(F_{t}\right)$, and $c_{0}\left(F_{t}\right)$, respectively; that is,

$$
\begin{aligned}
& {\left[\ell_{\infty}\left(F_{t}\right)\right]_{\mathscr{B}(\widehat{r}, \widehat{s})}=\left\{u^{*}=\left(u_{\left(t_{1}, t_{2}\right)}^{k}\right)\right.} \\
& \left.\quad \in w(F): \mathscr{B}(\widehat{r}, \widehat{s}) u_{\left(t_{1}, t_{2}\right)}^{k} \in \ell_{\infty}\left(F_{t}\right)\right\} \\
& {\left[c\left(F_{t}\right)\right]_{\mathscr{B}(\widehat{r}, \widehat{s})}=\left\{u^{*}=\left(u_{\left(t_{1}, t_{2}\right)}^{k}\right)\right.} \\
& \left.\quad \in w(F): \mathscr{B}(\widehat{r}, \widehat{s}) u_{\left(t_{1}, t_{2}\right)}^{k} \in c\left(F_{t}\right)\right\} \\
& {\left[c_{0}\left(F_{t}\right)\right]_{\mathscr{B}(\widehat{r}, \widehat{s})}=\left\{u^{*}=\left(u_{\left(t_{1}, t_{2}\right)}^{k}\right)\right.} \\
& \left.\quad \in w(F): \mathscr{B}(\widehat{r}, \widehat{s}) u_{\left(t_{1}, t_{2}\right)}^{k} \in c_{0}\left(F_{t}\right)\right\}
\end{aligned}
$$

We should emphasize here that the sequence spaces $\left[\ell_{\infty}\left(F_{t}\right)\right]_{\mathscr{B}(\hat{r}, \hat{s})},\left[c\left(F_{t}\right)\right]_{\mathscr{B}(\hat{r}, \hat{s})}$, and $\left[c_{0}\left(F_{t}\right)\right]_{\mathscr{B}(\hat{r}, \hat{s})}$ of triangular fuzzy numbers can be reduced to the sets $\ell_{\infty}\left(F_{t}\right), c\left(F_{t}\right)$, and $c_{0}\left(F_{t}\right)$, respectively, in the case $r^{k}=1, s^{k}=0$ for all $k \in \mathbb{N}$, in the structure of generalized difference matrix. So, the 
properties and results related to the sequence spaces of $\left[\ell_{\infty}\left(F_{t}\right)\right]_{\mathscr{B}(\hat{r}, \hat{s})},\left[c\left(F_{t}\right)\right]_{\mathscr{B}(\hat{r}, \hat{s})}$, and $\left[c_{0}\left(F_{t}\right)\right]_{\mathscr{B}(\hat{r}, \hat{s})}$ are more general and more extensive than the corresponding consequences of the spaces $\ell_{\infty}\left(F_{t}\right), c\left(F_{t}\right)$, and $c_{0}\left(F_{t}\right)$, respectively.

Let us define the sequence of fuzzy numbers $y=\left(y_{\left(t_{1}, t_{2}\right)}^{k}\right)$ which will be constantly used as the $\mathscr{B}(\widehat{r}, \widehat{s})$-transform of a sequence of fuzzy numbers $u^{*}=\left(u_{\left(t_{1}, t_{2}\right)}^{k}\right)$; that is,

$$
y_{\left(t_{1}, t_{2}\right)}^{k}=s^{k-1} u_{\left(t_{1}, t_{2}\right)}^{k-1}+r^{k} u_{\left(t_{1}, t_{2}\right)}^{k} \text {, }
$$

where $u_{\left(t_{1}, t_{2}\right)}^{-1}=\theta, r^{k}, s^{k} \in \mathbb{R}-\{0\}$ for all $k \in \mathbb{N}$.

Now, we may begin with the following theorem which is essential in the text.

Theorem 4. The sequence spaces $\left[\ell_{\infty}\left(F_{t}\right)\right]_{\mathscr{B}(\widehat{r}, \hat{s})},\left[c\left(F_{t}\right)\right]_{\mathscr{B}(\widehat{r}, \widehat{s})}$, and $\left[c_{0}\left(F_{t}\right)\right]_{\mathscr{B}(\hat{r}, \hat{s})}$ are linearly isomorphic to the spaces $\ell_{\infty}\left(F_{t}\right)$, $c\left(F_{t}\right)$, and $c_{0}\left(F_{t}\right)$, respectively; that is, $\left[\ell_{\infty}\left(F_{t}\right)\right]_{\mathscr{B}(\widehat{r}, \widehat{s})} \cong \ell_{\infty}\left(F_{t}\right)$, $\left[c\left(F_{t}\right)\right]_{\mathscr{B}(\hat{r}, \widehat{s})} \cong c\left(F_{t}\right)$, and $\left[c_{0}\left(F_{t}\right)\right]_{\mathscr{B}(\widehat{r}, \widehat{s})} \cong c_{0}\left(F_{t}\right)$.

Proof. Since the others can be similarly proved, we consider only the case $\left[\ell_{\infty}\left(F_{t}\right)\right]_{\mathscr{R}(\hat{r}, \widehat{s})} \cong \ell_{\infty}\left(F_{t}\right)$. To prove this, we should show the existence of a linear bijection between the spaces $\left[\ell_{\infty}\left(F_{t}\right)\right]_{\mathscr{B}(\hat{r}, \hat{s})}$ and $\ell_{\infty}\left(F_{t}\right)$. Consider the transformation defined $T$, with the notation of $(20)$, from $\left[\ell_{\infty}\left(F_{t}\right)\right]_{\mathscr{B}(\widehat{r}, \widehat{s})}$ to $\ell_{\infty}\left(F_{t}\right)$ by $u^{*} \mapsto y^{*}=T u^{*}=s^{k-1} u_{\left(t_{1}, t_{2}\right)}^{k-1}+r^{k} u_{\left(t_{1}, t_{2}\right)}^{k}$. The equality $T\left(u^{*}+w^{*}\right)=T u^{*}+T w^{*}$, where $u^{*}, w^{*} \epsilon$ $\left[\ell_{\infty}\left(F_{t}\right)\right]_{\mathscr{B}(\hat{r}, \hat{s})}$, is clear. Let us suppose that $\alpha \in \mathbb{R}$; then,

$$
\begin{aligned}
T\left(\alpha u^{*}\right) & =s^{k-1}\left(\alpha u_{\left(t_{1}, t_{2}\right)}^{k-1}\right)+r^{k}\left(\alpha u_{\left(t_{1}, t_{2}\right)}^{k}\right) \\
& =\alpha\left[s^{k-1} u_{\left(t_{1}, t_{2}\right)}^{k-1}+r^{k} u_{\left(t_{1}, t_{2}\right)}^{k}\right]=\alpha T u^{*} .
\end{aligned}
$$

That is, $T$ has the property homogeneity. Thus, $T$ is linear.

Let us take any $y^{*} \in \ell_{\infty}\left(F_{t}\right)$ and represent the sequence $u^{*}$ using $\mathscr{B}^{-1}(\widehat{r}, \widehat{s})$ as follows:

$$
u^{*}=\left(u_{\left(t_{1}, t_{2}\right)}^{k}\right)=\left(\mathfrak{B}^{k} y_{\left(t_{1}, t_{2}\right)}^{k}\right)
$$

where

$$
\begin{aligned}
\mathfrak{B}^{k} & =\sum_{n=0}^{k} \frac{(-1)^{k-n}}{r^{k}} \prod_{j=n}^{k-1} \frac{s^{j}}{r^{j}}, \\
\mathfrak{B}^{k-1} & =\sum_{n=0}^{k-1} \frac{(-1)^{k-n}}{r^{k}} \prod_{j=n}^{k-2} \frac{s^{j}}{r^{j}} .
\end{aligned}
$$

Then, we have

$$
\begin{aligned}
& \left\|u^{*}\right\|_{\left[\ell_{\infty}\left(F_{t}\right)\right]_{\mathscr{B}(\hat{r}, s)}}=\sup _{k} \bar{d}\left(\mathscr{B}(\widehat{r}, \widehat{s}) u_{\left(t_{1}, t_{2}\right)}^{k}, \theta\right) \\
& =\sup _{k} \bar{d}\left(s^{k-1} u_{\left(t_{1}, t_{2}\right)}^{k-1}+r^{k} u_{\left(t_{1}, t_{2}\right)}^{k}, \theta\right) \\
& =\sup _{k \in \mathbb{N}} \bar{d}\left(s^{k-1}\left[\mathfrak{B}^{k-1} y_{\left(t_{1}, t_{2}\right)}^{k-1-j}\right]+r^{k}\left[\mathfrak{B}^{k} y_{\left(t_{1}, t_{2}\right)}^{k-j}\right], \theta\right) \\
& =\sup _{k} \bar{d}\left(y_{\left(t_{1}, t_{2}\right)}^{k}, \theta\right)=\left\|y^{*}\right\|_{\ell_{\infty}\left(F_{t}\right)} .
\end{aligned}
$$

That is, $T$ is norm preserving. Consequently, the spaces $\left[\ell_{\infty}\left(F_{t}\right)\right]_{\mathscr{R}(\hat{r}, \hat{s})}$ and $\ell_{\infty}\left(F_{t}\right)$ are linearly isomorphic. It is clear here that if the spaces $\left[\ell_{\infty}\left(F_{t}\right)\right]_{\mathscr{B}(\hat{r}, \hat{s})}$ and $\ell_{\infty}\left(F_{t}\right)$ are, respectively, replaced by the spaces $\left[c\left(F_{t}\right)\right]_{\mathscr{B}(\hat{r}, \widehat{s})}$ and $c\left(F_{t}\right),\left[c_{0}\left(F_{t}\right)\right]_{\mathscr{B}(\hat{r}, \hat{s})}$ and $c_{0}\left(F_{t}\right)$, then we obtain the fact that $\left[c\left(F_{t}\right)\right]_{\mathscr{B}(\widehat{r}, \hat{s})} \cong c\left(F_{t}\right)$ and $\left[c_{0}\left(F_{t}\right)\right]_{\mathscr{B}(\hat{r}, \widehat{s})} \cong c_{0}\left(F_{t}\right)$. This completes the proof.

Theorem 5. The sets $\left[c\left(F_{t}\right)\right]_{\mathscr{B}(\widehat{r}, \widehat{s})}, \quad\left[c_{0}\left(F_{t}\right)\right]_{\mathscr{B}(\widehat{r}, \widehat{s})}$, and $\left[\ell_{\infty}\left(F_{t}\right)\right]_{\mathscr{B}(\hat{r}, \hat{s})}$ are complete normed sequence space of the triangular fuzzy numbers with the norm defined by

$$
\begin{gathered}
\left\|u^{*}\right\|=\sup _{k} \max \left\{\mathscr{B}(\widehat{r}, \widehat{s})\left|u_{\left(t_{1}, t_{2}\right)}^{k}-y_{\left(t_{1}, t_{2}\right)}^{k}-t_{1}\right|, \mathscr{B}(\widehat{r}, \widehat{s})\right. \\
\left.\cdot\left|u_{\left(t_{1}, t_{2}\right)}^{k}-y_{\left(t_{1}, t_{2}\right)}^{k}\right|, \mathscr{B}(\widehat{r}, \widehat{s})\left|u_{\left(t_{1}, t_{2}\right)}^{k}-y_{\left(t_{1}, t_{2}\right)}^{k}+t_{2}\right|\right\} .
\end{gathered}
$$

Proof. It was seen that, in Theorem 4, the sequence spaces of triangular fuzzy numbers $\left[\ell_{\infty}\left(F_{t}\right)\right]_{\mathscr{B}(\hat{r}, \widehat{s})},\left[c\left(F_{t}\right)\right]_{\mathscr{B}(\hat{r}, \widehat{s})}$, and $\left[c_{0}\left(F_{t}\right)\right]_{\mathscr{B}(\hat{r}, \widehat{s})}$ are linearly isomorphic to the spaces $\ell_{\infty}\left(F_{t}\right)$, $c\left(F_{t}\right)$, and $c_{0}\left(F_{t}\right)$, respectively. Additionally, since the matrix $\mathscr{B}(\widehat{r}, \widehat{s})$ is normal (see, [15]) and $\ell_{\infty}\left(F_{t}\right), c\left(F_{t}\right)$, and $c_{0}\left(F_{t}\right)$ are complete module spaces, it is clear that the sequence spaces $\left[\ell_{\infty}\left(F_{t}\right)\right]_{\mathscr{B}(\hat{r}, \hat{s})},\left[c\left(F_{t}\right)\right]_{\mathscr{B}(\hat{r}, \widehat{s})}$, and $\left[c_{0}\left(F_{t}\right)\right]_{\mathscr{B}(\hat{r}, \hat{s})}$ are complete normed spaces with the norm defined in (25).

Theorem 6. Let $\left(u_{\left(t_{1}, t_{2}\right)}^{k}\right)$ be a sequence of triangular fuzzy numbers. If $\bar{d}\left(u_{\left(t_{1}, t_{2}\right)}^{k}, u_{\left(t_{1}, t_{2}\right)}\right) \rightarrow 0$ as $k \rightarrow \infty$, then $\bar{d}\left(s^{k-1} u_{\left(t_{1}, t_{2}\right)}^{k-1}+r^{k} u_{\left(t_{1}, t_{2}\right)}^{k}, u_{\left(t_{1}, t_{2}\right)}\right) \rightarrow 0$ as $k \rightarrow \infty$. It means that the matrix $\mathscr{B}(\widehat{r}, \widehat{s})$ is regular.

Proof. Let $\left(u_{\left(t_{1}, t_{2}\right)}^{k}\right)$ be a sequence of fuzzy numbers and $\bar{d}\left(u_{\left(t_{1}, t_{2}\right)}^{k}, u_{\left(t_{1}, t_{2}\right)}\right) \rightarrow 0$ as $k \rightarrow \infty$. Then for a given $\epsilon>0$ there exists a positive integer $n_{0}$ such that

$$
\bar{d}\left(u_{\left(t_{1}, t_{2}\right)}^{k}, u_{\left(t_{1}, t_{2}\right)}\right)<\frac{\epsilon}{2 M} \quad \text { for } k \geq n_{0}
$$

Therefore, we can write

$$
\begin{aligned}
\bar{d} & \left(\mathscr{B}(\widehat{r}, \widehat{s}) u_{\left(t_{1}, t_{2}\right)}^{k}, u_{\left(t_{1}, t_{2}\right)}\right) \\
& =\bar{d}\left(s^{k-1} u_{\left(t_{1}, t_{2}\right)}^{k-1}+r^{k} u_{\left(t_{1}, t_{2}\right)}^{k}, u_{\left(t_{1}, t_{2}\right)}\right) \\
& \leq s^{k-1} \bar{d}\left(u_{\left(t_{1}, t_{2}\right)}^{k-1}, u_{\left(t_{1}, t_{2}\right)}\right)+r^{k} \bar{d}\left(u_{\left(t_{1}, t_{2}\right)}^{k}, u_{\left(t_{1}, t_{2}\right)}\right) .
\end{aligned}
$$

If we take $M=\sup _{k}\left\{s^{k}, r^{k}\right\}$, then we see that $\bar{d}(\mathscr{B}(\widehat{r}$, $\left.\widehat{s}) u_{\left(t_{1}, t_{2}\right)}^{k}, u_{\left(t_{1}, t_{2}\right)}\right)<\epsilon$. That is,

$$
\lim _{k}\left(s^{k-1} u_{\left(t_{1}, t_{2}\right)}^{k-1}+r^{k} u_{\left(t_{1}, t_{2}\right)}^{k}\right)=u_{\left(t_{1}, t_{2}\right)}
$$

holds. This completes the proof.

Theorem 7. The inclusions $c\left(F_{t}\right) \subset\left[c\left(F_{t}\right)\right]_{\mathscr{B}(\widehat{r}, \widehat{s})}$ and $c_{0}\left(F_{t}\right) \subset$ $\left[c_{0}\left(F_{t}\right)\right]_{\mathscr{B}(\hat{r}, \widehat{s})}$ strictly hold.

Proof. To prove the validity of the inclusion $c_{0}\left(F_{t}\right) \quad C$ $\left[c_{0}\left(F_{t}\right)\right]_{\mathscr{B}(\hat{r}, \widehat{s})}$, let us take any $v^{*} \in c_{0}\left(F_{t}\right)$. Then, bearing in mind the regularity of the method $\mathscr{B}(\widehat{r}, \widehat{s})$ (see, Theorem 6 ), 
we immediately observe that $\mathscr{B}(\widehat{r}, \widehat{s}) v^{*} \in c_{0}\left(F_{t}\right)$ which means that $v^{*} \in\left[c_{0}\left(F_{t}\right)\right]_{\mathscr{B}(\hat{r}, \hat{s})}$. Hence, the inclusion $c_{0}\left(F_{t}\right) \subset$ $\left[c_{0}\left(F_{t}\right)\right]_{\mathscr{B}(\hat{r}, \hat{s})}$ holds. One can see by analogy that the strict inclusion $c\left(F_{t}\right) \subset\left[c\left(F_{t}\right)\right]_{\mathscr{B}(\hat{r}, \hat{s})}$ also holds. This completes the proof.

Let $\lambda\left(F_{t}\right), \mu\left(F_{t}\right) \subset w(F)$ and $\mathscr{A}=\left(a_{n k}\right)$ be an infinite matrix of fuzzy numbers and consider the following expressions:

$$
\begin{aligned}
\sup _{n} \sum_{k} \bar{d}\left(a_{n k}, \theta\right) & <\infty, \\
\sup _{n} \sum_{k}\left[\bar{d}\left(a_{n k}, \theta\right)\right]^{q} & <\infty, \\
\lim _{n} \bar{d}\left(a_{n k}, a^{k}\right) & =0, \quad \text { where }\left(a_{(0,0)}^{k}\right) \in w\left(F_{t}\right), \\
\lim _{n} \sum_{k} \bar{d}\left(a_{n k}, \theta\right) & =0, \\
\lim _{n} \sum_{k} a_{n k} & =1_{\left(t_{1}, t_{2}\right)}, \\
\lim _{n} a_{n k} & =\theta, \quad k \in \mathbb{N} .
\end{aligned}
$$

In [16], some matrix classes are characterized by Talo and Başar which are given in the following lemma.

Lemma 8 (see [16]). The following statements hold:

(12) $\mathscr{A} \in\left(\ell_{\infty}(F): \ell_{\infty}(F)\right), \mathscr{A} \in\left(c(F): \ell_{\infty}(F)\right), \mathscr{A} \in$ $\left(c_{0}(F): \ell_{\infty}(F)\right)$ if and only if (29) holds.

(13) $\mathscr{A} \in\left(\ell_{\infty}(F): c_{0}(F)\right)$ if and only if (32) holds.

(14) $\mathscr{A} \in\left(c_{0}(F): c(F)\right)$ if and only if (29) and (31) hold.

(15) $\mathscr{A} \in\left(c_{0}(F): c_{0}(F)\right)$ if and only if (29) and (31) hold with $a^{k}=\theta$ for all $k \in \mathbb{N}$.

(16) $\mathscr{A} \in\left(\ell_{p}(F): c(F)\right)$ if and only if (30) and (31) hold.

(17) $\mathscr{A} \in\left(\ell_{p}(F): c_{0}(F)\right)$ if and only if (30) and (31) hold with $a^{k}=\theta$ for all $k \in \mathbb{N}$.

(18) $\mathscr{A} \in(c(F): c(F), p)$ if and only if (29), (33), and (31) hold with $a^{k}=\theta$ for all $k \in \mathbb{N}$.

Analogously to Talo and Başar, we can prove the following propositions.

Proposition 9. $A \in\left(c\left(F_{t}\right): c\left(F_{t}\right)\right)$ if and only if (29) and (31) also hold with $a^{k}=\theta$ for all $k \in \mathbb{N}$.

\section{Real Duals of the Spaces $\left[\ell_{\infty}\left(F_{t}\right)\right]_{\mathscr{B}(\hat{r}, \hat{s})}$, $\left[c\left(F_{t}\right)\right]_{\mathscr{B}(\widehat{r}, \widehat{s})}$, and $\left[c_{0}\left(F_{t}\right)\right]_{\mathscr{B}(\hat{r}, \widehat{s})}$}

In this section, we state and prove the theorems determining the $\beta(r)$ - and $\gamma(r)$-duals of the spaces $\left[\ell_{\infty}\left(F_{t}\right)\right]_{\mathscr{B}(\hat{r}, \widehat{s})}$,
$\left[c\left(F_{t}\right)\right]_{\mathscr{B}(\hat{r}, \hat{s})}$, and $\left[c_{0}\left(F_{t}\right)\right]_{\mathscr{B}(\hat{r}, \hat{s})}$. For the sequence spaces $\lambda\left(F_{t}\right)$ and $\mu\left(F_{t}\right)$, define the set $S\left(\lambda\left(F_{t}\right), \mu\left(F_{t}\right)\right)$ by

$$
\begin{aligned}
& S\left(\lambda\left(F_{t}\right), \mu\left(F_{t}\right)\right)=\left\{a^{*}=\left(a_{(0,0)}^{k}\right)=\left(a^{k}\right)\right. \\
& \left.\quad \in w:\left(a^{k} x_{\left(t_{1}, t_{2}\right)}^{k}\right) \in \mu\left(F_{t}\right) \forall x \in \lambda\left(F_{t}\right)\right\},
\end{aligned}
$$

where $w$ denotes all real valued sequences space. With the notation of (35), $\beta(r)$ - and $\gamma(r)$-duals of a sequence space $\lambda\left(F_{t}\right)$, which are respectively denoted by $\lambda^{\beta(r)}\left(F_{t}\right)$ and $\lambda^{\gamma(r)}\left(F_{t}\right)$, are defined by $\lambda^{\beta(r)}\left(F_{t}\right)=S\left(\lambda\left(F_{t}\right), c s\left(F_{t}\right)\right)$, and $\lambda^{\gamma(r)}\left(F_{t}\right)=S\left(\lambda\left(F_{t}\right), b s\left(F_{t}\right)\right)$. We will use a technique, in the proof of Theorems 10 and 12, which is used in [17-19].

Theorem 10. The $\gamma(r)$-dual of the spaces $\left[\ell_{\infty}\left(F_{t}\right)\right]_{\mathscr{B}(\hat{r}, \hat{s})}$, $\left[c\left(F_{t}\right)\right]_{\mathscr{B}(\hat{r}, \widehat{s})}$, and $\left[c_{0}\left(F_{t}\right)\right]_{\mathscr{B}(\hat{r}, \widehat{S})}$ is the set

$$
\begin{aligned}
D_{1} & =\left\{a^{*}=\left(a_{(0,0)}^{k}\right)=\left(a^{k}\right)\right. \\
& \left.\in w: \sup _{n} \sum_{k=0}^{n} \bar{d}\left(\sum_{i=k}^{n} \frac{(-1)^{i}}{r^{i}} \prod_{j=k}^{i-1} \frac{s^{j}}{r^{j}} a^{i}, \theta\right)<\infty\right\} .
\end{aligned}
$$

Proof. Since the proof is similar for the rest of the spaces, we determine only $\gamma(r)$-dual of the set $\left[c_{0}\left(F_{t}\right)\right]_{\mathscr{B}(\hat{r}, \hat{S})}$. Let $a^{*} \in$ $w(\mathbb{R})$ and define the matrix $\mathscr{Z}=\left(z_{n k}\right)$ via the sequence $\left(a^{i}\right)$ by

$$
z_{n k}= \begin{cases}\sum_{i=k}^{n} \frac{(-1)^{i}}{r^{i}} \prod_{j=k}^{i-1} \frac{s^{j}}{r^{j}} a^{i}, & (0 \leq k \leq n) \\ 0, & (k>n)\end{cases}
$$

$$
(k, j, n \in \mathbb{N}) .
$$

Bearing in mind relation (20) we immediately derive that

$$
\begin{aligned}
\sum_{k=0}^{n} a^{k} x_{\left(t_{1}, t_{2}\right)}^{k} & =\sum_{k=0}^{n}\left(\sum_{j=0}^{k} \frac{(-1)^{k-j}}{r^{k}} \prod_{i=j}^{k-1} \frac{s^{i}}{r^{i}} y^{j}\right) a^{k} \\
& =\left(\mathscr{X} y_{\left(t_{1}, t_{2}\right)}\right)^{n}, \quad(k, j, n \in \mathbb{N}) .
\end{aligned}
$$

From (38) we realize that $a^{*} x=\left(a^{k} x_{\left(t_{1}, t_{2}\right)}^{k}\right) \in b s\left(F_{t}\right)$ whenever $x \in\left[c_{0}\left(F_{t}\right)\right]_{\mathscr{B}(\hat{r}, \hat{s})}$ if and only if $\mathscr{Z} y_{\left(t_{1}, t_{2}\right)} \in \ell_{\infty}\left(F_{t}\right)$ whenever $y_{\left(t_{1}, t_{2}\right)} \in c_{0}\left(F_{t}\right)$. Then, by considering Part (12) of Lemma 8, we have

$$
\sup _{n} \sum_{k=0}^{n} \bar{d}\left(\sum_{i=k}^{n} \frac{(-1)^{i}}{r^{i}} \prod_{j=k}^{i-1} \frac{s^{j}}{r^{j}} a^{i}, \theta\right)<\infty,
$$

which yields the consequence that $\left[c_{0}\left(F_{t}\right)\right]_{\mathscr{B}(\tilde{r}, \hat{s})}^{\gamma(r)}=D_{1}$.

Lemma 11 (see [20], Theorem 3.1). Let $\mathscr{C}=\left(c_{n k}\right)$ be defined via a sequence $a^{*}=\left(a^{k}\right) \in$ wand the inverse matrix $\mathscr{V}=\left(v_{n k}\right)$ of the triangle matrix $\mathscr{U}=\left(u_{n k}\right)$ by

$$
c_{n k}= \begin{cases}\sum_{j=k}^{n} a^{j} v_{j k}, & 0 \leq k \leq n, \\ 0, & k>n,\end{cases}
$$


for all $k, n \in \mathbb{N}$. Then

$$
\begin{aligned}
& \left\{\lambda_{\mathcal{U}}\right\}^{\gamma}=\left\{a^{*}=\left(a^{k}\right) \in w: \mathscr{C} \in\left(\lambda: \ell_{\infty}\right)\right\}, \\
& \left\{\lambda_{\mathcal{U}}\right\}^{\beta}=\left\{a^{*}=\left(a^{k}\right) \in w: \mathscr{C} \in(\lambda: c)\right\} .
\end{aligned}
$$

Theorem 12. Define the sets $D_{2}$ and $D_{3}$ by

$$
\begin{aligned}
D_{2} & =\left\{a^{*} \in w: \lim _{n} \bar{d}\left(\sum_{k=0}^{n} \frac{(-1)^{n-k}}{r^{n}} \prod_{j=k}^{n-1} \frac{s^{j}}{r^{j}} a^{n}, \theta\right)=0\right\}, \\
D_{3} & =\left\{a^{*} \in w: \lim _{n} \bar{d}\left(\sum_{k=0}^{n} \frac{(-1)^{n-k}}{r^{n}} \prod_{j=k}^{n-1} \frac{s^{j}}{r^{j}} a^{n}, a^{k}\right)\right. \\
& \left.=0 \text {, where }\left(a_{(0,0)}^{k}\right) \in w\left(F_{t}\right)\right\} .
\end{aligned}
$$

Then, $\left[c_{0}\left(F_{t}\right)\right]_{\mathscr{B}(\hat{r}, \widehat{s})}^{\beta(r)}=D_{1} \cap D_{3}$. And $\left[c\left(F_{t}\right)\right]_{\mathscr{B}(\widehat{r}, \widehat{s})}^{\beta(r)}=D_{1} \cap D_{2}$.

Proof. It is clear from Lemmas 8 and 11.

\section{Matrix Transformations}

For the first time, Lorentz introduced the concept of dual summability methods for the limitation which depends on a Stieltjes integral and passed to the discontinuous matrix methods by means of a suitable step function [21]. Later, many authors, such as Başar [22], Başar and Çolak [23], Kuttner [24], Lorentz and Zeller [25], and Şengönül and Başar [19] worked on the dual summability methods.

Let us suppose that the set $\left[\lambda\left(F_{t}\right)\right]_{\mathscr{B}(\hat{r}, \widehat{s})}$ is any of the sets $\left[c_{0}\left(F_{t}\right)\right]_{\mathscr{B}(\hat{r}, \widehat{s})},\left[c\left(F_{t}\right)\right]_{\mathscr{B}(\hat{r}, \widehat{s})}$, and $\left[\ell_{\infty}\left(F_{t}\right)\right]_{\mathscr{B}(\hat{r}, \widehat{s})}$. In this section, we characterize the matrix mappings from $\left[\lambda\left(F_{t}\right)\right]_{\mathscr{B}(\hat{r}, \widehat{s})}$ into any given sequence space of triangular fuzzy numbers via the concept of the dual summability methods and vice versa, so we call it the sequential generalized difference dual summability methods. Let us suppose that the sequences $u^{*}=$ $\left(u_{\left(t_{1}, t_{2}\right)}^{i}\right)$ and $v^{*}=\left(v_{\left(t_{1}, t_{2}\right)}^{i}\right)$ are connected with (20) and let $\mathscr{A}$ transform of the sequence $u^{*}=\left(u_{\left(t_{1}, t_{2}\right)}^{i}\right)$ be $z^{*}=\left(z_{\left(t_{1}, t_{2}\right)}^{i}\right)$ and let the $\mathscr{B}$-transform of the sequence $v^{*}=\left(v_{\left(t_{1}, t_{2}\right)}^{i}\right)$ be $t^{*}=\left(t_{\left(t_{1}, t_{2}\right)}^{i}\right)$; that is,

$$
\begin{aligned}
& z_{\left(t_{1}, t_{2}\right)}^{i}=\left(\mathscr{A} u_{\left(t_{1}, t_{2}\right)}\right)^{i}=\sum_{i} a_{n i} u_{\left(t_{1}, t_{2}\right)}^{i}, \quad(i \in \mathbb{N}), \\
& t_{\left(t_{1}, t_{2}\right)}^{i}=\left(\mathscr{B} v_{\left(t_{1}, t_{2}\right)}\right)^{i}=\sum_{i} b_{n i} v_{\left(t_{1}, t_{2}\right)}^{i}, \quad(i \in \mathbb{N}) .
\end{aligned}
$$

It is clear here that the method $\mathscr{B}$ is applied to the $\mathscr{B}(\widehat{r}, \widehat{s})$ transform $v^{*}=\left(v_{\left(t_{1}, t_{2}\right)}^{i}\right)=\left\{(\mathscr{B}(\widehat{r}, \widehat{s}) u)^{i}\right\}$ of the sequence $u^{*}=$ $\left(u_{\left(t_{1}, t_{2}\right)}^{i}\right)$ while the method $\mathscr{A}$ is directly applied to the terms of the sequence $u^{*}=\left(u_{\left(t_{1}, t_{2}\right)}^{i}\right)$. So, the methods $\mathscr{A}$ and $\mathscr{B}$ are essentially different (see, [22]).

Let us assume the existence of the matrix product $\mathscr{B} \mathscr{B}(\widehat{r}$, $\widehat{s})$ which is a much weaker assumption than the conditions on the matrix $\mathscr{B}$ belonging to any matrix class, in general. If $z_{\left(t_{1}, t_{2}\right)}^{i}$ becomes $t_{\left(t_{1}, t_{2}\right)}^{i}$ (or $t_{\left(t_{1}, t_{2}\right)}^{i}$ becomes $\left.z_{\left(t_{1}, t_{2}\right)}^{i}\right)$, under the application of the formal summation by parts, then the methods $\mathscr{A}$ and $\mathscr{B}$ in (43) are called sequential generalized difference dual type matrices. This leads us to the fact that $\mathscr{B} \mathscr{B}(\widehat{r}, \widehat{s})$ exists and is equal to $\mathscr{A}$ and $\mathscr{A}=$ $(\mathscr{B} \mathscr{B}(\widehat{r}, \widehat{s})) u_{\left(t_{1}, t_{2}\right)}^{i}=\mathscr{B}\left(\mathscr{B}(\widehat{r}, \widehat{s}) u_{\left(t_{1}, t_{2}\right)}^{i}\right)=\mathscr{B} v$ formally holds. This statement is equivalent to the following relation between the elements of the matrices $\mathscr{A}=\left(a_{n i}\right)$ and $\mathscr{B}=\left(b_{n i}\right)$ :

$$
\begin{aligned}
b_{n i} & =\sum_{k=i}^{\infty} \frac{(-1)^{k}}{r^{k}} \prod_{j=i}^{k-1} \frac{s^{j}}{r^{j}} a_{n k} \\
\text { or } a_{n i} & =r^{i} b_{n i}+s^{i} b_{n, i+1}
\end{aligned}
$$

for all $n, i \in \mathbb{N}$. Furthermore one can see that easily $v_{\left(t_{1}, t_{2}\right)}^{i}$ reduces $u_{\left(t_{1}, t_{2}\right)}^{i}$, as in the following:

$$
v_{\left(t_{1}, t_{2}\right)}^{i}=\sum_{i} b_{n i} y^{i}=\sum_{i} b_{n i}\left(r^{i} x_{i}+s^{i-1} x^{i-1}\right)=u_{\left(t_{1}, t_{2}\right)}^{i}
$$

Now we may give the following theorem concerning to the sequential generalized difference dual matrices.

Theorem 13. Let $\mathscr{A}=\left(a_{n i}\right)$ and $\mathscr{B}=\left(b_{n i}\right)$ be the sequential generalized difference dual type matrices and let $\mu\left(F_{t}\right)$ be any given sequence space. Then, $\mathscr{A} \in\left(\left[c\left(F_{t}\right)\right]_{\mathscr{B}(\hat{r}, \widehat{s})}: \mu\left(F_{t}\right)\right)$ if and only if $\left(a_{n i}\right)_{k \in \mathbb{N}} \in \ell_{1}\left(F_{t}\right)$ and $\mathscr{B} \in\left(c\left(F_{t}\right): \mu\left(F_{t}\right)\right)$.

Proof. Suppose that $\mathscr{A}=\left(a_{n i}\right)$ and $\mathscr{B}=\left(b_{n i}\right)$ are sequential generalized difference dual type matrices which means that (44) holds. Additionally, let $\mu\left(F_{t}\right)$ be any given sequence space and take into account that the spaces $\left[c\left(F_{t}\right)\right]_{\mathscr{B}(\hat{r}, \widehat{s})}$ and $c\left(F_{t}\right)$ are linearly isomorphic.

Let $\mathscr{A} \in\left(\left[c\left(F_{t}\right)\right]_{\mathscr{B}(\widehat{r}, \widehat{s})}: \mu\left(F_{t}\right)\right)$ and take any $u^{*} \in c\left(F_{t}\right)$. Then, $\mathscr{B} \mathscr{B}(\widehat{r}, \widehat{s})$ is equal to $\mathscr{A}$ and $\left(a_{n i}\right)_{i \in \mathbb{N}} \in D_{1} \cap D_{4} \cap D_{5}=$ $\left[c\left(F_{t}\right)\right]_{\mathscr{B}(\widehat{r}, \widehat{s})}^{\beta(r)}$ which yields that $\left(b_{n i}\right)_{k \in \mathbb{N}} \in \ell_{1}\left(F_{t}\right)$ for each $n \in$ $\mathbb{N}$. Hence $\mathscr{B} u^{*}$ exists for each $u^{*} \in c\left(F_{t}\right)$ and we have the following equation;

$$
\sum_{i} b_{n i} u_{\left(t_{1}, t_{2}\right)}^{i}=\sum_{i} a_{n i} v_{\left(t_{1}, t_{2}\right)}^{i} \quad(n \in \mathbb{N}) .
$$

Subsequently, it is clear from (44) that $\mathscr{B} u^{*}=\mathscr{A} v^{*}$ which leads us to the consequence that $\mathscr{B} \in\left(c\left(F_{t}\right): \mu\left(F_{t}\right)\right)$.

Conversely, suppose that $\mathscr{B} \in\left(\left[c\left(F_{t}\right)\right]_{\mathscr{B}(\hat{r}, \widehat{s})}: \mu\left(F_{t}\right)\right)$ and take any $v^{*} \in\left[c\left(F_{t}\right)\right]_{\mathscr{B}(\widehat{r}, \widehat{s})}$. Then, $\mathscr{A} v^{*}$ exists. Therefore, we obtain from the following equality as $n \rightarrow \infty$ that $\mathscr{A} v^{*}=$ $\mathscr{B} u^{*}$ and this shows that $\mathscr{A} \in\left(\left[c\left(F_{t}\right)\right]_{\mathscr{B}(\hat{r}, \widehat{s})}: \mu\left(F_{t}\right)\right)$ :

$$
\sum_{i=0}^{n} a_{n i} v_{\left(t_{1}, t_{2}\right)}^{i}=\sum_{i=0}^{n}\left\{\sum_{k=i}^{n} \frac{(-1)^{k}}{r^{i}} \prod_{j=i}^{k-1} \frac{s^{j}}{r^{j}} a_{n i}\right\} u_{\left(t_{1}, t_{2}\right)}^{i}
$$

$(n \in \mathbb{N})$

This completes the proof. 
Theorem 14. Suppose that the elements of the infinite matrices $\mathscr{D}=\left(d_{n i}\right)$ and $\mathscr{E}=\left(e_{n i}\right)$ are connected with the relation

$$
e_{n i}=s^{n-1} d_{n-1, i}+r^{n} d_{n i}, \quad(n, i \in \mathbb{N})
$$

and $\mu\left(F_{t}\right)$ is any given sequence space. Then, $\mathscr{D} \in\left(\mu\left(F_{t}\right)\right.$ : $\left.\left[c\left(F_{t}\right)\right]_{\mathscr{B}(\widehat{r}, \widehat{s})}\right)$ if and only if $\mathscr{E} \in\left(\mu\left(F_{t}\right): c\left(F_{t}\right)\right)$.

Proof. Let $u^{*} \in \mu\left(F_{t}\right)$ and consider the following equality:

$$
\begin{aligned}
& \left\{\mathscr{B}(\widehat{r}, \widehat{s})\left(\mathscr{D} u_{\left(t_{1}, t_{2}\right)}\right)\right\}^{n} \\
& =s\left(\mathscr{D} u_{\left(t_{1}, t_{2}\right)}\right)^{n-1}+r\left(\mathscr{D} u_{\left(t_{1}, t_{2}\right)}\right)^{n} \\
& =s^{n-1} \sum_{i} d_{n-1, i} u_{\left(t_{1}, t_{2}\right)}^{i}+r^{n} \sum_{i} d_{n i} u_{\left(t_{1}, t_{2}\right)}^{i} \\
& \quad=\sum_{i}\left(s^{n-1} d_{n-1, i}+r^{n} d_{n i}\right) u_{\left(t_{1}, t_{2}\right)}^{i}=\left(\mathscr{E} u_{\left(t_{1}, t_{2}\right)}\right)^{n},
\end{aligned}
$$

$(n, i \in \mathbb{N})$

Which yields that $\mathscr{D} u^{*} \in\left[c\left(F_{t}\right)\right]_{\mathscr{B}(r, s)}$ if and only if $\mathscr{E} u^{*} \epsilon$ $c\left(F_{t}\right)$. This step completes the proof.

Now, right here, we give the following propositions which are obtained from Lemma 8 and Theorems 13 and 14 .

Proposition 15. Let $\mathscr{A}=\left(a_{n i}\right)$ be an infinite matrix of real numbers. Then one has the following:

(1) $\mathscr{A}=\left(a_{n i}\right) \in\left(\left[\ell_{\infty}\left(F_{t}\right)\right]_{\mathscr{B}(\widehat{r}, \widehat{s})}: \ell_{\infty}\left(F_{t}\right)\right)$ if and only if $\left(a_{n i}\right)_{i \in \mathbb{N}} \in\left[\ell_{\infty}\left(F_{t}\right)\right]_{\mathscr{B}(\hat{r}, \widehat{s})}^{\beta(r)}$ for each $n \in \mathbb{N}$ and

$$
\sup _{n} \sum_{k=0}^{n} \bar{d}\left(\sum_{i=k}^{n} \frac{(-1)^{n-i}}{r^{i}} \prod_{j=k}^{i-1} \frac{s^{j}}{r^{j}} a_{n i}, \theta\right)<\infty
$$

holds for all $i \in \mathbb{N}$.

(2) $\mathscr{A}=\left(a_{n i}\right) \in\left(\left[c\left(F_{t}\right)\right]_{\mathscr{B}(\hat{r}, \widehat{s})}: c\left(F_{t}\right)\right)$ if and only if (50) holds and $\left(a_{n i}\right)_{i \in \mathbb{N}} \in\left[c\left(F_{t}\right)\right]_{\mathscr{B}(\widehat{r}, \widehat{s})}^{\beta(r)}$ also holds for all $n \in$ $\mathbb{N}$ and

$$
\lim _{n} \bar{d}\left(\sum_{k=0}^{n} \frac{(-1)^{n-k}}{r^{n}} \prod_{j=k}^{n-1} \frac{s^{j}}{r^{j}} a_{n j}, \theta\right)=0 .
$$

Proposition 16. Let $\mathscr{A}=\left(a_{n i}\right)$ be an infinite matrix of real numbers. Then, $\mathscr{A}=\left(a_{n i}\right) \in\left(\ell_{\infty}\left(F_{t}\right):\left[\ell_{\infty}\left(F_{t}\right)\right]_{\mathscr{B}(\hat{r}, \widehat{s})}\right)$ if and only if

$$
\sup _{n} \sum_{k=0}^{\infty} \bar{d}\left(s^{k-1} a_{n, k-1}+r^{k} a_{n k}, \theta\right)<\infty .
$$

Proposition 17. Let $\mathscr{A}=\left(a_{n i}\right)$ be an infinite matrix of real numbers. Then, $\mathscr{A}=\left(a_{n i}\right) \in\left(c\left(F_{t}\right):\left[c\left(F_{t}\right)\right]_{\mathscr{B}(\widehat{r}, \hat{s})}\right)$ if and only if

$$
\lim _{n} \bar{d}\left(s^{k-1} a_{n, k-1}+r^{k} a_{n k}, u_{\left(t_{1}, t_{2}\right)}^{k}\right)=0
$$

with $u_{\left(t_{1}, t_{2}\right)}^{k} \in F_{t}$ and $k \in \mathbb{N}$ holds.

\section{Determining the Center of Gravity of Sequence Space of Triangular Fuzzy Numbers and Similarity Measures between Fuzzy Numbers}

In this section, we give the COG points of a sequence space of triangular fuzzy numbers by means of [7]. Let us suppose that $\left(u_{\left(t_{1}, t_{2}\right)}^{k}\right)$ is a sequence of triangular fuzzy number. Then, the values $x_{u^{k}}^{\star}$ and $y_{u^{k}}^{\star}$ of the COG points of $\left(u_{\left(t_{1}, t_{2}\right)}^{k}\right)$ are presented as follows:

$$
\begin{aligned}
y_{u^{k}}^{\star} & =\lim _{k} \frac{w_{u^{k}} \times\left(\left(u_{2}^{k}-u_{2}^{k}\right) /\left(u_{3}^{k}-u_{1}^{k}\right)+2\right)}{6}=\frac{w_{u^{k}}}{3} \\
& =\frac{1}{3}, \\
x_{u^{k}}^{\star} & =\lim _{k} \frac{y_{u^{k}}^{\star}\left(2 u_{2}^{k}\right)+\left(u_{3}^{k}+u_{1}^{k}\right)\left(w_{u^{k}}-y_{u^{k}}^{\star}\right)}{2 w_{u^{k}}} .
\end{aligned}
$$

Based on (54) and (55), we can determine the COG points of a sequence of triangular fuzzy number $\left(u_{\left(t_{1}, t_{2}\right)}^{k}\right)$ as $\left(x_{u^{k}}^{\star}, y_{u^{k}}^{\star}\right)$.

Let us suppose that $\mathbf{u}$ and $\mathbf{v}$ are two classical fuzzy numbers where $\mathbf{u}=\left(\mathbf{u}_{1}, \mathbf{u}_{2}, \mathbf{u}_{3}\right)$ and $\mathbf{v}=\left(\mathbf{v}_{1}, \mathbf{v}_{2}, \mathbf{v}_{3}\right)$; then the degree of similarity $S$ between $\mathbf{u}$ and $\mathbf{v}$ is calculated by [6] as in the following:

$$
S(\mathbf{u}, \mathbf{v})=1-\frac{\sum_{i=1}^{3}\left|\mathbf{u}_{i}-\mathbf{v}_{i}\right|}{3},
$$

where $S(\mathbf{u}, \mathbf{v}) \in[0,1]$.

In [5], Lee proposed a similarity measure for classical fuzzy numbers and used the similarity measure to deal with fuzzy opinions for group decision making, where the degree of similarity $S(\mathbf{u}, \mathbf{v})$ between the triangular fuzzy numbers $\mathbf{u}$ and $\mathbf{v}$ can be calculated as follows:

$$
\begin{aligned}
S(\mathbf{u}, \mathbf{v})=1-\frac{\|\mathbf{u}-\mathbf{v}\|_{\ell_{p}}}{\|U\|} \times 3^{-1 / p} & \\
\|\mathbf{u}-\mathbf{v}\|_{\ell_{p}} & =\left(\sum_{i=1}^{3}\left(\left|\mathbf{u}_{i}-\mathbf{v}_{i}\right|\right)^{p}\right)^{1 / p}
\end{aligned}
$$

and $\|U\|=\max (U)-\min (U)$ where $U$ is the universe of discourse. The larger the value of $S$, the higher the similarity between the components of $U$.

In [4], Hsieh and Chen defined a similarity measure method where the degree of similarity $S(\mathbf{u}, \mathbf{v})$ between classical fuzzy numbers $\mathbf{u}$ and $\mathbf{v}$ can be given as in the following:

$$
\begin{aligned}
S(\mathbf{u}, \mathbf{v})= & \frac{1}{1+d(\mathbf{u}, \mathbf{v})}, \\
& d(\mathbf{u}, \mathbf{v})=\left|\frac{\mathbf{u}_{1}+4 \mathbf{u}_{2}+\mathbf{u}_{3}}{6}-\frac{\mathbf{v}_{1}+4 \mathbf{v}_{2}+\mathbf{v}_{3}}{6}\right|,
\end{aligned}
$$

where $\left(\mathbf{u}_{1}+4 \mathbf{u}_{2}+\mathbf{u}_{3}\right) / 6$ and $\left(\mathbf{v}_{1}+4 \mathbf{v}_{2}+\mathbf{v}_{3}\right) / 6$ represent graded mean integration components of $\mathbf{u}$ and $\mathbf{v}$, respectively.

In addition to these similarity measure methods, S.-J. Chen and S.-M. Chen [7] introduced a simple center of 
gravity method represented as SCGM, to determine the center of gravity point of a generalized fuzzy number by using the concept of geometry. Besides, they gave a method to evaluate the degree of similarity between generalized fuzzy numbers.

In the present paper we generalize these formulas to sequence spaces of triangular fuzzy numbers. By this method, we will fill the big gap in the literature.

\section{Similarity Measures between Sequence Spaces of Triangular Fuzzy Numbers}

Assume that there are two sequences of triangular fuzzy numbers, $u^{*}=\left(u^{k}-t_{1}, u^{k}, u^{k}+t_{2}\right)$ and $v^{*}=\left(v^{k}-t_{1}, v^{k}, v^{k}+t_{2}\right)$, $t_{1}, t_{2} \in \mathbb{R}, t_{1} \leq t_{2}$; then the degree of similarity $S\left(u^{*}, v^{*}\right)$ between the sequences of triangular fuzzy numbers $u^{*}$ and $v^{*}$ can be calculated as in the following:

$$
\begin{aligned}
& S\left(u^{*}, v^{*}\right) \\
& \quad=\frac{\inf \left\{h\left(u^{*}\right), h\left(v^{*}\right)\right\}}{\sup \left\{h\left(u^{*}\right), h\left(v^{*}\right)\right\}}\left[1-\frac{1}{3} \lim _{k} \sum_{n=1}^{3}\left|u_{n}^{k}-v_{n}^{k}\right|\right] \\
& \quad=\alpha,
\end{aligned}
$$

where $S\left(u^{*}, v^{*}\right) \in[0,1]$ and the notion of $h\left(u^{*}\right)$ shows the highest membership degree of fuzzy number $u^{k}$. For all $k \in \mathbb{N}, h\left(u^{*}\right)$ is considered as 1 because of the validity of fuzzy number conditions, through all the text. The function $S: w(F) \times w(F) \rightarrow \mathbb{R}$ is called similarity degree between sequences of fuzzy sets $u^{*}$ and $v^{*}$. If $S\left(u^{*}, v^{*}\right)=1$, then we say that $u^{*}$ is completely similar to the sequence $v^{*}$; if $0<S\left(u^{*}, v^{*}\right)=\alpha<1$, then we say that the sequence $u^{*}$ is $\alpha$-similar to the sequence $v^{*}$; if $\alpha \leq 0$ we can say that $u^{*}$ is not similar to $v^{*}$.

Now, we introduce another similarity measure method for sequence of triangular fuzzy numbers as follows:

$$
\begin{gathered}
S\left(u^{*}, v^{*}\right)=1-\frac{\left\|u^{*}-v^{*}\right\|_{\ell_{p}}}{\|w(U)\|} \times 3^{-1 / p} \\
\left\|u^{*}-v^{*}\right\|_{\ell_{p}}=\left(\lim _{k} \sum_{n=1}^{3}\left(\left|u_{n}^{k}-v_{n}^{k}\right|\right)^{p}\right)^{1 / p}, \\
\|w(U)\|=\sup (w(U))-\inf (w(U)) .
\end{gathered}
$$

We take $p=1, t_{1}=0, t_{2}=0.6$ for examples, through all the text.

One of the other similarity measure methods for sequence of triangular fuzzy numbers is given as follows:

$$
\begin{aligned}
& S\left(u^{*}, v^{*}\right)=\frac{1}{1+d\left(u^{*}, v^{*}\right)}, \\
& d\left(u^{*}, v^{*}\right)=\lim _{k}\left|\frac{u^{k}-t_{1}+4 u^{k}+u^{k}+t_{2}}{6}-\frac{v^{k}-t_{1}+4 v^{k}+v^{k}+t_{2}}{6}\right| .
\end{aligned}
$$

In [7], S.-J. Chen and S.-M. Chen introduced a simple center of gravity method to determine the center of gravity point of a generalized fuzzy number by using the concept of geometry. By means of this method, we will generalize this formula to the sequence of fuzzy numbers as follows:

$$
\begin{aligned}
& S\left(u^{*}, v^{*}\right)=\lim _{k}\left(1-\frac{\sum_{i=1}^{3}\left|u_{i}^{k}-v_{i}^{k}\right|}{3}\right) \\
& \cdot \frac{\inf \left\{h\left(u^{*}\right), h\left(v^{*}\right)\right\}}{\sup \left\{h\left(u^{*}\right), h\left(v^{*}\right)\right\}}\left(1-\left|x_{u^{k}}^{\star}-x_{v^{k}}^{\star}\right|\right)^{B\left(S_{u^{*}}, S_{v^{*}}\right)},
\end{aligned}
$$

where $x_{u^{k}}^{\star}$ and $x_{v^{k}}^{\star}$ are calculated by (55) and $h\left(u^{*}\right)=h\left(v^{*}\right)=$ 1 since the fuzzy number property and $B\left(S_{u^{*}}, S_{v^{*}}\right)$ are defined as follows:

$$
B\left(S_{u^{*}}, S_{v^{*}}\right)= \begin{cases}(1), & \text { if } \lim _{k}\left(S_{u^{*}}+S_{v^{*}}\right)>0 \\ (0), & \text { if } \lim _{k}\left(S_{u^{*}}+S_{v^{*}}\right)=0,\end{cases}
$$

where $S_{u^{*}}$ and $S_{v^{*}}$ are the lengths of the bases of the $k$ th place of the sequence of triangular fuzzy numbers $u^{*}$ and $v^{*}$, respectively, defined as follows by means of [7]:

$$
S_{u^{*}}=u^{k}+t_{2}-\left(u^{k}-t_{1}\right)=t_{2}+t_{1} .
$$

Next, we deal with three sets of sequence spaces of triangular fuzzy numbers to compare similarity measures mentioned above. The calculation results are listed in Table 1. Now, we write the equations of three sets of sequences of triangular fuzzy numbers as in the following:

$$
\begin{aligned}
& \text { SET I: }\left(t_{1}=0, t_{2}=0.6\right) \\
& \left(u_{1}^{k}\right) \\
& =\left(\frac{k}{10(k+1)}-t_{1}, \frac{k}{10(k+1)}, \frac{k}{10(k+1)}+t_{2}\right),
\end{aligned}
$$

$\left(u_{1}^{k}\right)$

$$
=\left(\frac{-k}{10(k+1)}-t_{1}, \frac{-k}{10(k+1)}, \frac{-k}{10(k+1)}+t_{2}\right) .
$$

SET II: $\left(t_{1}=0, t_{2}=0.6\right)$

$$
\begin{aligned}
& \left(u_{1}^{k}\right) \\
& =\left(\frac{k}{10(k+1)}-t_{1}, \frac{k}{10(k+1)}, \frac{k}{10(k+1)}+t_{2}\right), \\
& \left(u_{2}^{k}\right) \\
& =\left(\frac{4 k}{10(k+1)}-t_{1}, \frac{4 k}{10(k+1)}, \frac{4 k}{10(k+1)}+t_{2}\right) . \\
& \text { SET III: }\left(t_{1}=0, t_{2}=0\right) \\
& \left(u_{1}^{k}\right)=\left(0.2-t_{1}, 0.2,0.2+t_{2}\right) \text {, } \\
& \left(u_{2}^{k}\right)=\left(0.3-t_{1}, 0.3,0.3+t_{2}\right) \text {. }
\end{aligned}
$$

We can give a comparison of the computing conclusions of the similarity measures of the above equations with the methods mentioned above as in Table 1. 
TABLE 1: A comparison between similarity measure methods.

\begin{tabular}{lccc}
\hline \multirow{2}{*}{ Similarity measures } & \multicolumn{3}{c}{ Sets of sequence of } \\
& triangular fuzzy numbers \\
& SET I & SET II & SET III \\
\hline Lee's Method [5] & 0.333 & 0.166 & 0.5 \\
Chen and Lin's Method [6] & 0.6 & 0.5 & 0.9 \\
Hsieh and Chen's Method [4] & 0.833 & 0.769 & 0.909 \\
Chen and Chen's Method [7] & 0.48 & 0.35 & 0.54 \\
\hline
\end{tabular}

TABLE 2: Linguistic values of $W_{i}^{k}$ and $R_{i}^{k}$ of the subcomponents $A_{1}^{k}$, $A_{2}^{k}$, and $A_{3}^{k}$.

\begin{tabular}{lcc}
\hline $\begin{array}{l}\text { Subcomponents } \\
\left(A_{i}^{k}\right)\end{array}$ & $\begin{array}{c}\text { Linguistic values of } \\
\text { severity of loss }\left(W_{i}^{k}\right)\end{array}$ & $\begin{array}{c}\text { Linguistic values of } \\
\text { probability of failure } \\
\left(R_{i}^{k}\right)\end{array}$ \\
\hline$A_{1}^{k}$ & Low & Low \\
$A_{2}^{k}$ & Fairly-high & Medium \\
$A_{3}^{k}$ & Very-low & High \\
\hline
\end{tabular}

\section{Fuzzy Risk Analysis Based on the Similarity Measure of Sequence of Triangular Fuzzy Numbers}

Fuzzy weighted mean method is used in $[26,27]$ for introducing fuzzy risk analysis. According to [26] for every $i$, subcomponent $A_{i}$ is measured by two evaluating items represented as $R_{i}$ and $W_{i}$ that denote the probability of failure of the subcomponents $A_{i}$ and severity of loss of the subcomponents $A_{i}$, respectively, and $1 \leq i \leq 3$. Kangari and Riggs [26] and Schmucker [27] use the linguistic terms ("absolutely high," "fairly-high," "high," "medium," "low," "fairly-low," and "absolutely-low") for determining the values of $R_{i}$ and $W_{i}$. In [7], S.-J. Chen and S.-M. Chen use ninemember linguistic terms based on $[28,29]$ to represent the linguistic terms in their paper. We show the linguistic terms and sequence of triangular fuzzy numbers in Table 3 and generalize $R_{i}$ and $W_{i}$ to sequences $\left(R_{i}^{k}\right)$ and $\left(W_{i}^{k}\right)$, respectively. In addition to these, we present the algorithm for fuzzy sequence risk analysis by means of [7] as follows.

Step 1. Use the fuzzy weighted mean method to integrate the evaluating items $R_{i}^{k}$ and $W_{i}^{k}$ of sequence of each subcomponent $A_{i}^{k}$, where $1 \leq i \leq n$, to get the total risk $R^{*}$ of the sequence of component $A^{*}=\left(A^{k}\right)$ shown as follows:

$$
R^{*}=\lim _{k} \frac{\sum_{i=1}^{n}\left(W_{i}^{k} \otimes R_{i}^{k}\right)}{\sum_{i=1}^{n} W_{i}^{k}} .
$$

Step 2. Use the similarity measure (62) to measure the degree of similarity between the sequences of triangular fuzzy number $R^{*}$ and Table 3 includes each linguistic term used for classifying the sequence space of triangular fuzzy numbers.

In the following, we use an example presented in [27].

Example 18. Let us consider that the component $A^{k}$ consists of three subcomponents $A_{1}^{k}, A_{2}^{k}, A_{3}^{k}$, as shown in Figure 1.
TABLE 3: Linguistic terms and sequence of triangular fuzzy numbers.

\begin{tabular}{ll}
\hline $\begin{array}{l}\text { A nine-member linguistic term set } \\
\text { Linguistic terms }\end{array}$ & Sequence of triangular fuzzy numbers \\
\hline Absolutely-low & $\left(0-t_{1}, 0,0+t_{2}\right)$ \\
Very-low & $\left(\frac{2 k+100}{100 k}-t_{1}, \frac{2 k+100}{100 k}, \frac{2 k+100}{100 k}+t_{2}\right)$ \\
Low & $\left(\frac{36 k+100}{100 k}-t_{1}, \frac{18 k+100}{100 k}, \frac{18 k+100}{100 k}+t_{2}\right)$ \\
Fairly-low & $\left(\frac{58 k+100}{100 k}-t_{1}, \frac{58 k+100}{100 k}, \frac{58 k+100}{100 k}+t_{2}\right)$ \\
Medium & $\left(\frac{80 k+100}{100 k}-t_{1}, \frac{80 k+100}{100 k}, \frac{80 k+100}{100 k}+t_{2}\right)$ \\
Fairly-high & $\left(\frac{92 k+100}{100 k}-t_{1}, \frac{92 k+100}{100 k}, \frac{92 k+100}{100 k}+t_{2}\right)$ \\
High & $\left(\frac{98 k+100}{100 k}-t_{1}, \frac{98 k+100}{100 k}, \frac{98 k+100}{100 k}+t_{2}\right)$ \\
Very-high & $\left(1-t_{1}, 1,1+t_{2}\right)$ \\
Absolutely-high &
\end{tabular}

Now, we would like to measure the probability of failure $R^{*}$ of the component $A^{*}=\left(A^{k}\right)$. Table 2 shows the linguistic values of the two evaluating items $R_{i}^{k}$ and $W_{i}^{k}$, respectively, similar to [27], where the linguistic values are shown by sequence of triangular fuzzy numbers in Table 3.

In the following, we use the proposed fuzzy risk analysis method to consider the fuzzy risk analysis problem.

Step 1. By considering $\oplus, \otimes, \oslash,(68)$, and Tables 2 and 3 , the probability of failure $R^{*}=\left(R^{k}\right)$ of the component $A^{*}=\left(A^{k}\right)$ can be measured as in Table 3 .

Consider $R^{*}=\left(R^{k}\right)=\lim _{k}[$ low $\otimes$ low $\oplus$ medium $\otimes$ fairly-high $\oplus$ high $\otimes$ very-low $] \oslash[$ low $\oplus$ fairly-high $\oplus$ very-low $]$ $=\left(0.5148-t_{1}, 0.5148,0.5148+t_{2}\right)=(0.5148,0.5148,1.1148)$.

Step 2. We determine COG points of the nine-member linguistic term set shown in Table 4 by using (54) and (55) and by using (64) we obtain the base $S$ of all the sequences of triangular fuzzy numbers as $t_{2}+t_{1}$. In the same way, we can get the COG point of the sequence of triangular fuzzy number $R^{*}$, where $\operatorname{COG}\left(R^{*}\right)=\left(0.5096+\left(t_{2}-t_{1}\right) 0.33,0.33\right)=(0.7096,0.33)$. Based on (63) and Table 4, we can see that the values $B\left(S_{R^{*}}, S_{\text {absolutely-low }}\right), \quad B\left(S_{R^{*}}, S_{\text {very-low }}\right), \quad B\left(S_{R^{*}}, S_{\text {low }}\right), \quad B\left(S_{R^{*}}\right.$, $\left.S_{\text {fairly-low }}\right), B\left(S_{R^{*}}, S_{\text {medium }}\right), B\left(S_{R^{*}}, S_{\text {fairly-high }}\right), B\left(S_{R^{*}}, S_{\text {high }}\right)$, $B\left(S_{R^{*}}, S_{\text {very-high }}\right)$, and $B\left(S_{R^{*}}, S_{\text {absolutely-high }}\right)$ are equal to 1 . And $S_{\text {absolutely-low }}=S_{\text {very-low }}=S_{\text {low }}=S_{\text {fairly-low }}=S_{\text {medium }}=$ $S_{\text {fairly-high }}=S_{\text {high }}=S_{\text {very-high }}=S_{\text {absolutely-high }}=t_{2}+t_{1}=0.6$.

By using (62), the degree of similarity between the sequence of triangular fuzzy number $R^{*}=\left(R^{k}\right)$ and the linguistic terms shown in Table 2 can be evaluated as follows: $S\left(R^{*}\right.$, absolutely-low $)=0.1383, S\left(R^{*}\right.$, very-low $)=0.1541$, 


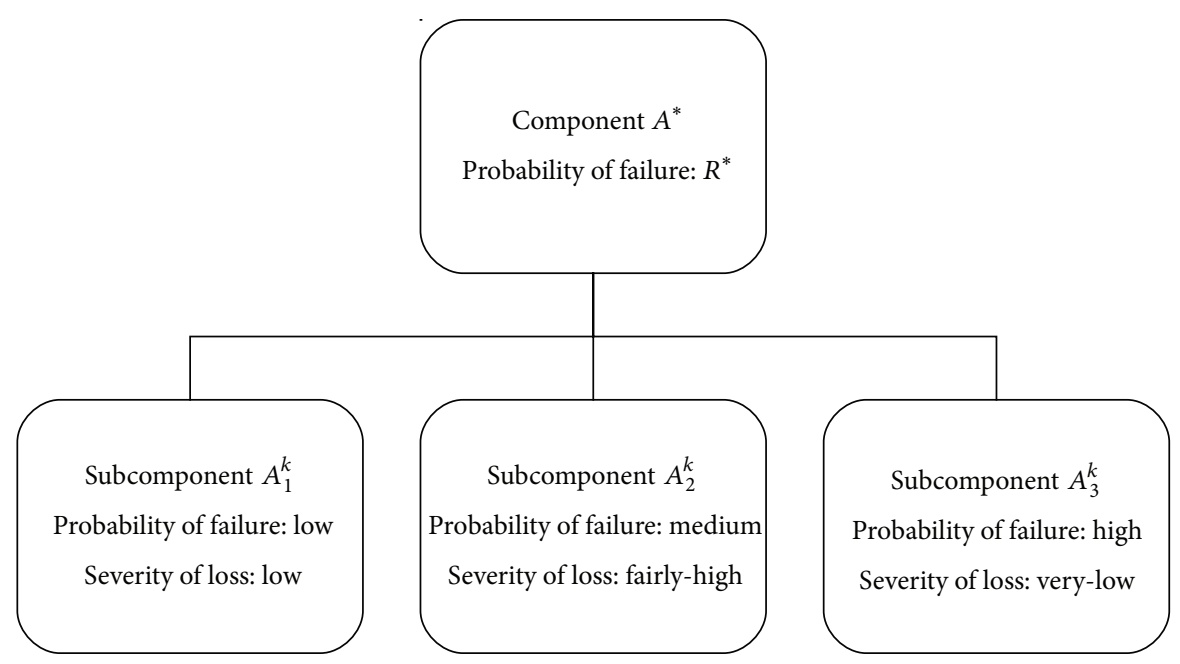

FIGURE 1: Structure of risk analysis.

TABLE 4: Linguistic terms and correspondence to COG Terms.

\begin{tabular}{|c|c|}
\hline \multicolumn{2}{|c|}{$\begin{array}{l}\text { The COG points of the } \\
\text { nine-member linguistic term set }\end{array}$} \\
\hline Linguistic terms & COG points \\
\hline Absolutely-low & COG $($ absolutely-low $)=(0.2,0.33)$ \\
\hline Very-low & COG $($ very-low $)=(0.22,0.33)$ \\
\hline Low & $\operatorname{COG}($ low $)=(0.38,0.33)$ \\
\hline Fairly-low & COG $($ fairly-low $)=(0.56,0.33)$ \\
\hline Medium & $\operatorname{COG}($ medium $)=(0.78,0.33)$ \\
\hline Fairly-high & $\operatorname{COG}($ fairly-high $)=(1,0.33)$ \\
\hline High & COG $($ high $)=(1.12,0.33)$ \\
\hline Very-high & COG $($ very-high $)=(1.18,0.33)$ \\
\hline Absolutely-high & COG $($ absolutely-high $)=(1.2,0.33)$ \\
\hline
\end{tabular}

$S\left(R^{*}\right.$, low $)=0.3094, S\left(R^{*}\right.$, fairly-low $)=0.5453, S\left(R^{*}\right.$, medium $)=0.7275, S\left(R^{*}\right.$, fairly-high $)=0.5038, S\left(R^{*}\right.$, high $)=0.3955, S\left(R^{*}\right.$, very-high $)=0.3449, S\left(R^{*}\right.$, absolutelyhigh $)=0.3285$. Because $S\left(R^{*}\right.$, medium $)=0.9018$ has the largest value, the sequence of triangular fuzzy number $R^{*}=$ $\left(R^{k}\right)$ is translated into the linguistic term "medium," where the degree of similarity is 0.9018 . That is, the probability of failure of the component $A^{*}=\left(A^{k}\right)$ is medium.

\section{Conflict of Interests}

The author declares that there is no conflict of interests regarding the publication of this paper.

\section{References}

[1] L. A. Zadeh, "Fuzzy sets," Information and Computation, vol. 8, pp. 338-353, 1965.

[2] M. Matloka, "Sequences of fuzzy numbers," Busefal, no. 28, pp. 28-37, 1986.

[3] S. Nanda, "On sequences of fuzzy numbers," Fuzzy Sets and Systems, vol. 33, no. 1, pp. 123-126, 1989.
[4] C. H. Hsieh and S. H. Chen, "Similarity of generalized fuzzy numbers with graded mean integration representation," in Proceedings of the 8th International Fuzzy Systems Association World Congress, vol. 2, pp. 551-555, Taipei, Taiwan, 1999.

[5] H.-S. Lee, "Optimal aggregation method for fuzzy opinions of group decision," in Proceedings of the IEEE International Conference on Systems, Man, and Cybernetics, vol. 3, pp. 314-319, October 1999.

[6] S.-M. Chen, "New methods for subjective mental workload assessment and fuzzy risk analysis," Cybernetics and Systems, vol. 27, no. 5, pp. 449-472, 1996.

[7] S.-J. Chen and S.-M. Chen, "Fuzzy risk analysis based on similarity measures of generalized fuzzy numbers," IEEE Transactions on Fuzzy Systems, vol. 11, no. 1, pp. 45-56, 2003.

[8] S.-M. Chen, "Evaluating the rate of aggregative risk in software development using fuzzy set theory," Cybernetics and Systems, vol. 30, no. 1, pp. 57-75, 1999.

[9] R. E. Moore, "Automatic error analysis in digital computation," Tech. Rep. LSMD-48421, Lockheed Missiles and Space Company, 1959.

[10] P. Diamond and P. Kloeden, Metric Spaces of Fuzzy Sets: Theory and Applications, World Scientific, Singapore, 1994.

[11] M. L. Puri and D. A. Ralescu, "Differentials of fuzzy functions," Journal of Mathematical Analysis and Applications, vol. 91, no. 2, pp. 552-558, 1983.

[12] M. Şengönül, "On the Zweier sequence spaces of fuzzy numbers," International Journal of Mathematics and Mathematical Sciences, vol. 2014, Article ID 439169, 9 pages, 2014.

[13] Z. Mitrovic and S. Rusov, "Z similarity measure among fuzzy sets," FME Transactions, no. 34, pp. 115-119, 2006.

[14] Z. Zararsız and M. Sengönül, "On the gravity of center of sequence of fuzzy numbers," Annals of Fuzzy Mathematics and Informatics, vol. 6, no. 3, pp. 479-485, 2013.

[15] A. Wilansky, Summability Through Functional Analysis, vol. 85 of North-Holland Mathematics Studies, North-Holland Publishing, Amsterdam, The Netherlands, 1984.

[16] Ö. Talo and F. Başar, "Determination of the duals of classical sets of sequences of fuzzy numbers and related matrix transformations," Computers \& Mathematics with Applications, vol. 58, no. 4, pp. 717-733, 2009. 
[17] B. Altay, F. Başar, and M. Mursaleen, "On the Euler sequence spaces which include the spaces $l_{p}$ and $l_{\infty}$," Information Sciences, vol. 176, no. 10, pp. 1450-1462, 2006.

[18] F. Başar and B. Altay, "On the space of sequences of $p$ bounded variation and related matrix mappings," Ukrainian Mathematical Journal, vol. 55, no. 1, pp. 136-147, 2003.

[19] M. Şengönül and F. Başar, "Some new Cesàro sequence spaces on non-absolute type which include the spaces $c_{0}$ and $c$," Soochow Journal of Mathematics, vol. 31, no. 1, pp. 107-119, 2005.

[20] B. Altay and F. Başar, "Certain topological properties and duals of the domain of a triangle matrix in a sequence space," Journal of Mathematical Analysis and Applications, vol. 336, no. 1, pp. 632-645, 2007.

[21] G. G. Lorentz, "Über Limitierungsverfahren, die von einem Stieltjes-Integral abhängen," Acta Mathematica, vol. 79, pp. 255272, 1947.

[22] F. Başar, "Matrix transformations between certain sequence spaces of $X_{p}$ and $l_{p}$," Soochow Journal of Mathematics, vol. 26, no. 2, pp. 191-204, 2000.

[23] F. Başar and R. Çolak, "Almost-conversative matrix transformations," Turkish Journal of Mathematics, vol. 13, no. 3, pp. 91-100, 1989.

[24] B. Kuttner, "On dual summability methods," Proceedings of the Cambridge Philosophical Society, vol. 71, pp. 67-73, 1972.

[25] G. G. Lorentz and K. Zeller, "Summation of sequences and summation of series," Proceedings of the Cambridge Philosophical Society, vol. 71, pp. 67-73, 1972.

[26] R. Kangari and L. S. Riggs, "Construction risk assessment by linguistics," IEEE Transactions on Engineering Management, vol. 36, no. 2, pp. 126-131, 1992.

[27] K. J. Schmucker, Fuzzy Sets, Natural Language Computations, and Risk Analysis, Computer Science, Rockville, Md, USA, 1984.

[28] S.-M. Chen, "New approach to handling fuzzy decisionmaking problems," IEEE Transactions on Systems, Man and Cybernetics, vol. 18, no. 6, pp. 1012-1016, 1988.

[29] W. R. Zhang, Knowledge representation using linguistic fuzzy relations [Ph.D. dissertation], University of South Carolina, Columbia, SC, USA, 1986. 


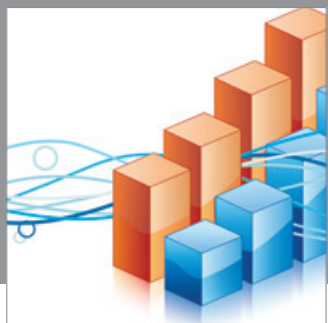

Advances in

Operations Research

mansans

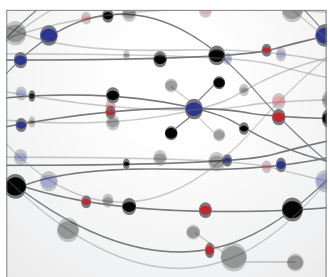

The Scientific World Journal
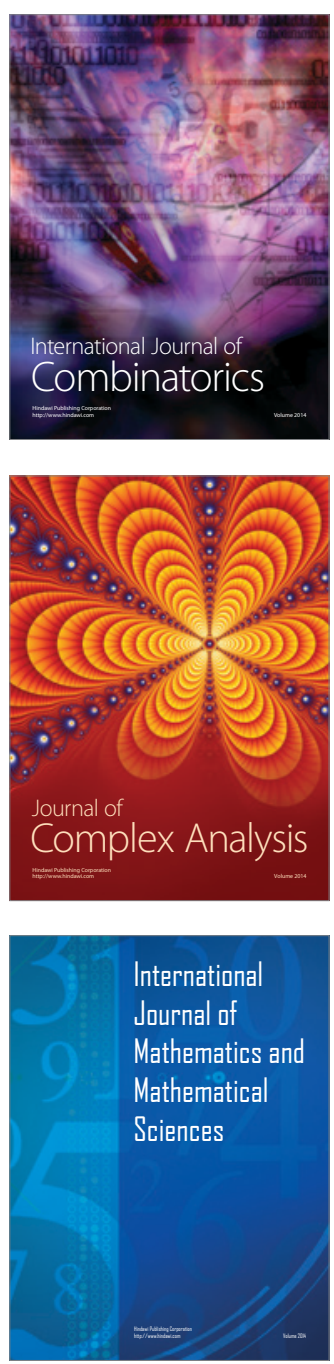
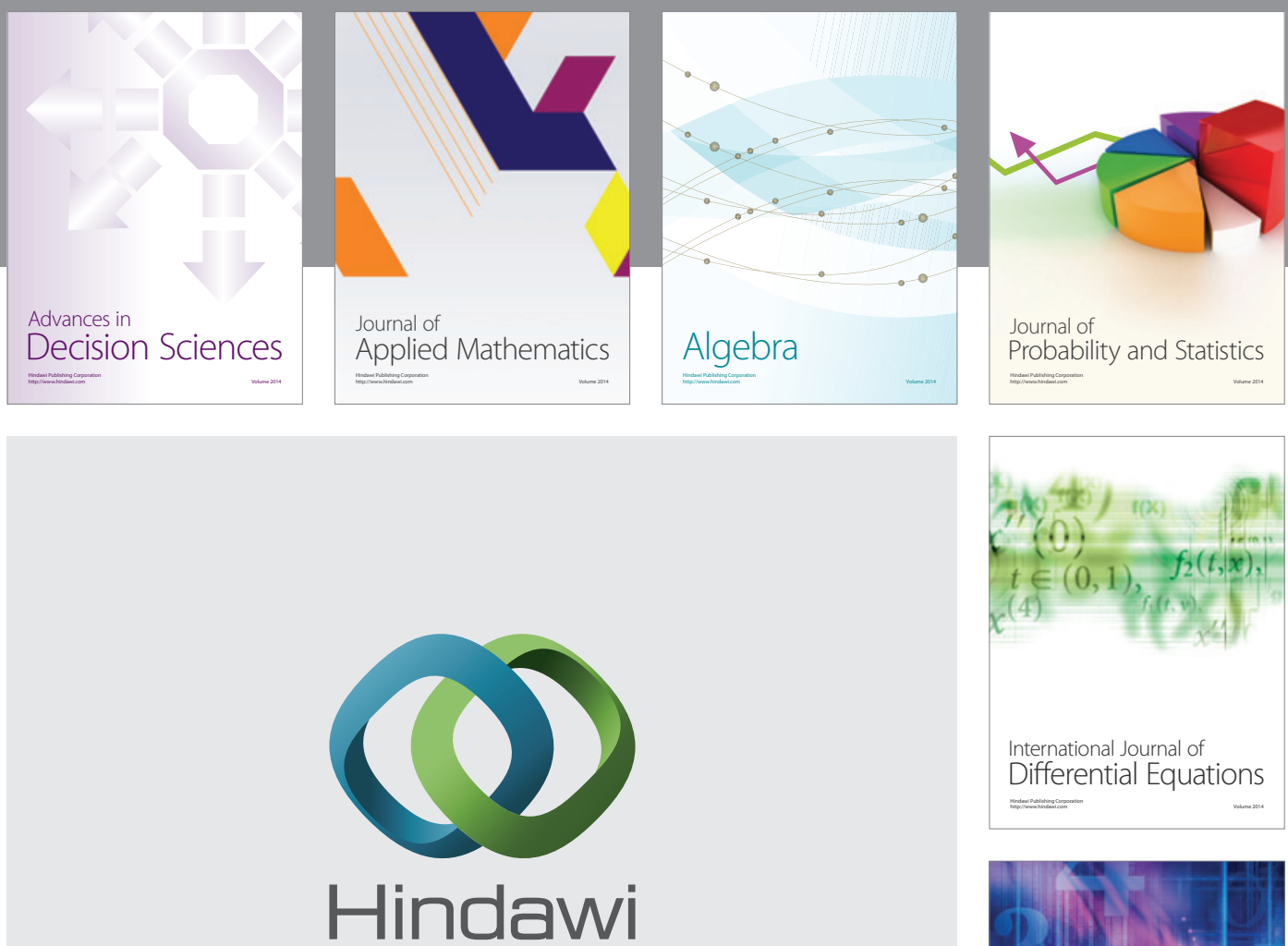

Submit your manuscripts at http://www.hindawi.com
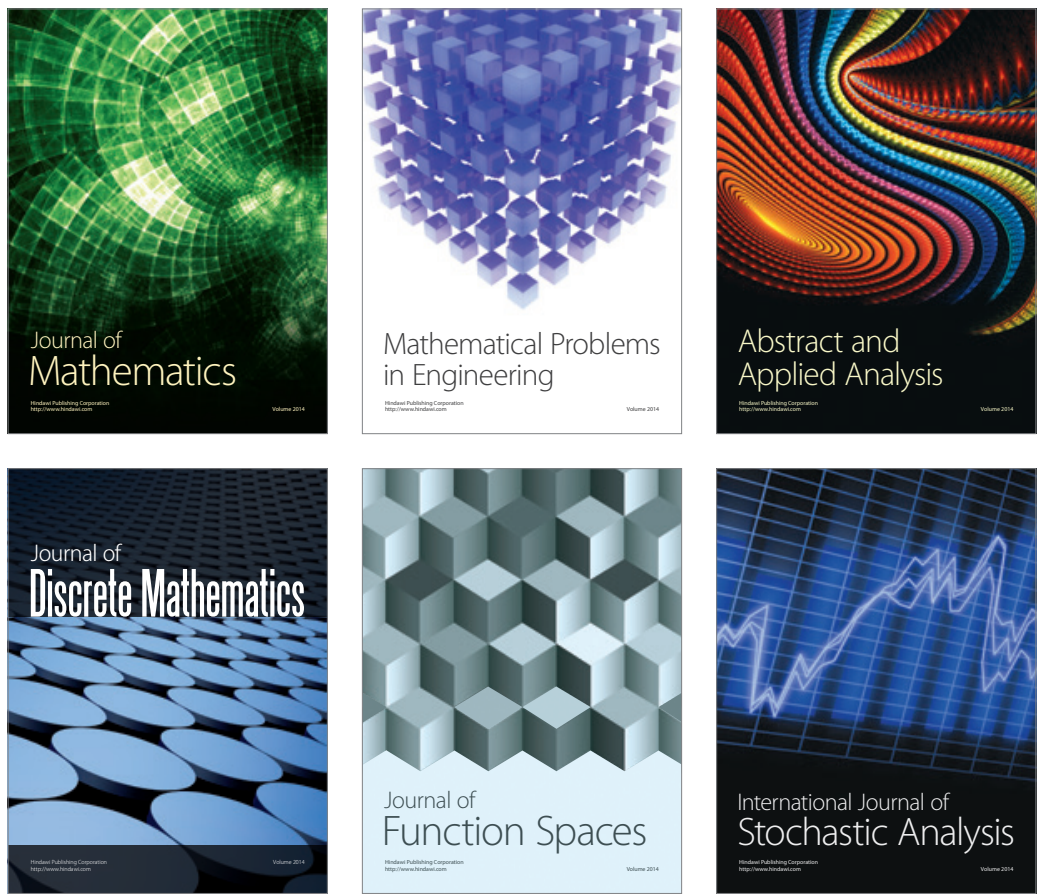

Journal of

Function Spaces

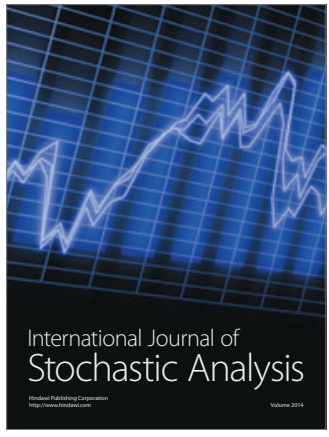

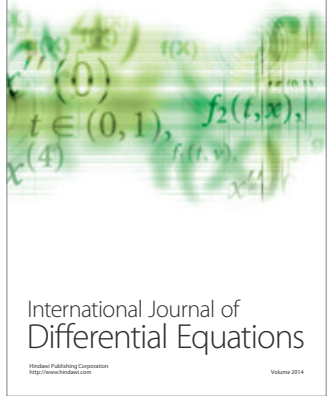
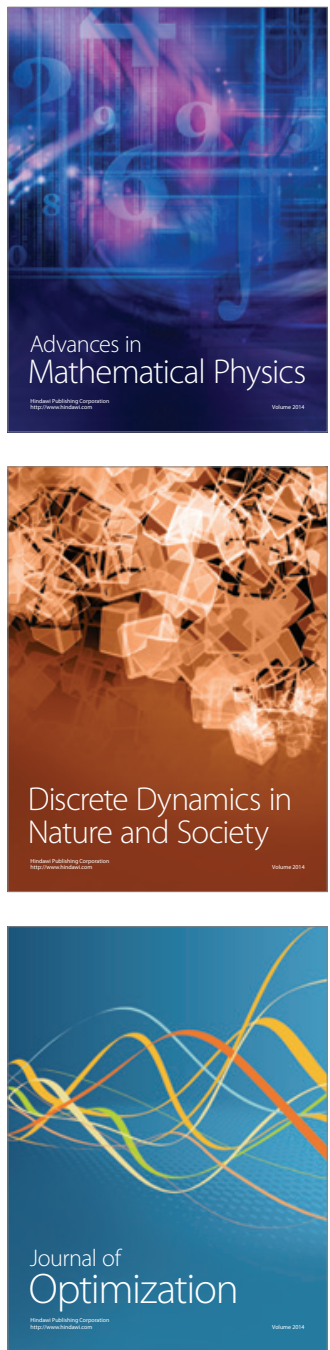